Portland State University

PDXScholar

6-15-2020

\title{
Follow the (Electronic) Money: How Bitcoin and Blockchain Technology Are "Shaking the System"
}

Jim Robert Mignano

Portland State University

Follow this and additional works at: https://pdxscholar.library.pdx.edu/open_access_etds

Part of the Political Science Commons

Let us know how access to this document benefits you.

Recommended Citation

Mignano, Jim Robert, "Follow the (Electronic) Money: How Bitcoin and Blockchain Technology Are "Shaking the System"'" (2020). Dissertations and Theses. Paper 5593.

https://doi.org/10.15760/etd.7465

This Thesis is brought to you for free and open access. It has been accepted for inclusion in Dissertations and Theses by an authorized administrator of PDXScholar. Please contact us if we can make this document more accessible: pdxscholar@pdx.edu. 
Follow the (Electronic) Money:

How Bitcoin and Blockchain Technology Are "Shaking the System"

by

Jim Robert Mignano

A thesis submitted in partial fulfillment of the requirements for the degree of

Master of Science

in

Political Science

Thesis Committee:

David Kinsella, Chair

Melody Ellis Valdini

Bruce Gilley

Portland State University

2020 


\section{(C) 2020 Jim Robert Mignano}

Creative Commons Attribution-ShareAlike 4.0 International (CC BY-SA 4.0)

$$
\text { (웅 (1) (2) }
$$




\begin{abstract}
Bitcoin is the first digital medium to allow global, "purely peer-to-peer" exchange. At the height of the Great Recession, Bitcoin's pseudonymous creator introduced the electronic cash to sidestep political and economic institutions. Today, it is praised as an opportunity for the unbanked, a liberating force, and a pioneering technology. It is also infamously associated with volatility, illicit activities, and profligate energy consumption.

Bitcoin has also flown under the radar of political science, whereas computer scientists, economists, and legal scholars have written extensively about it. To address the gap in the literature, I describe Bitcoin as an actor in global affairs, explain how Bitcoin and blockchain technology work, and discuss why Bitcoin is relevant to political science as the archetypal case of blockchain technology. I argue that Bitcoin is a form of contentious politics uniquely suited to the twenty-first century.

Examining Bitcoin as a form of contentious politics sheds light on how a purportedly borderless technology has actually fared in subverting state authority: not entirely well. Nonetheless, Bitcoin has succeeded in at least two respects: it is an unprecedented form of untraceable electronic cash that coordinates unrivaled levels of computing resources from voluntary contributors and it has become a resilient, global social movement. As an archetype of blockchain technology, Bitcoin inspires research of blockchain's capacity to facilitate collective action, uses in international cooperation and competition, and prospects for human development. The thesis concludes with a set of implications for blockchain research and policy.
\end{abstract}


Dedication

To my son, Jimmie. 


\section{Acknowledgments}

I am indebted to the members of my thesis committee for the valuable time and guidance they provided over the course of this project. Professor David Kinsella patiently embraced my explorations into this frontier topic, while guiding me to seek meaningful applications in international relations. Professor Melody Ellis Valdini helped situate my work as the foundation of a more comprehensive research agenda. Professor Bruce Gilley offered ample instructive critique of my work, strengthening it substantially.

I thank several members of Portland State University's faculty and staff for their instrumental help. Professor Nirupama Bulusu introduced me to blockchain's technical foundations. Professor Birol Yeşilada challenged me to think carefully about research design and Professor Lindsay Benstead instructed me in research methods. Kelly Mackura, Political Science Department Manager; Madison Powell, Political Science Operations Coordinator; Andrea Haack, Graduate Studies Specialist; and Sherry Buchanan, Digital Collections Technician, each played a role in facilitating this project.

I would also like to acknowledge several specific contributions to this project. Dr. Mary Jane Parmentier provided helpful suggestions for Chapter 1. Thom Linton developed the API used to compile blockchain data in Chapter 2. Andrew Bremner, Jeremy Baker, and Will Baker-who also supplied the Bitcoin ATM photo in Chapter 3catered to my numerous meanderings with crucial commentary. The accommodations of my supervisor, Pamela Deardorff, were indispensable. My family and friends were generous with support and encouragement. I am especially grateful for the precious time and patience devoted by my wife, Alicia, and son, Jimmie, as I undertook this project. 


\section{Table of Contents}

Abstract

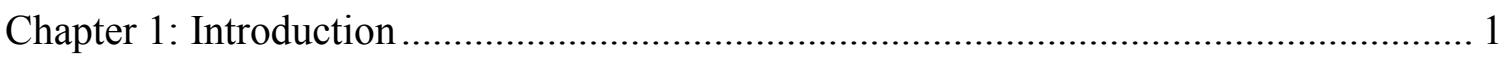

Chapter 2: What Is Bitcoin? .................................................................................. 4

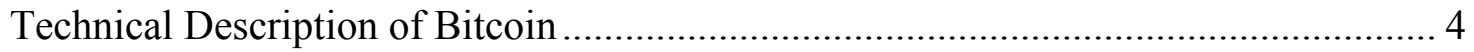

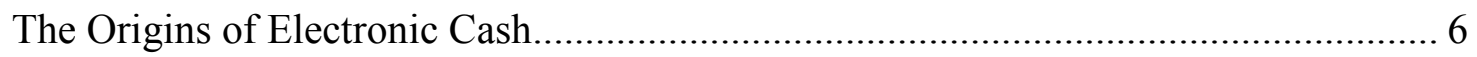

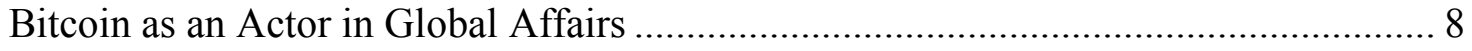

Implications of Bitcoin as an Actor in Global Affairs .............................................. 29

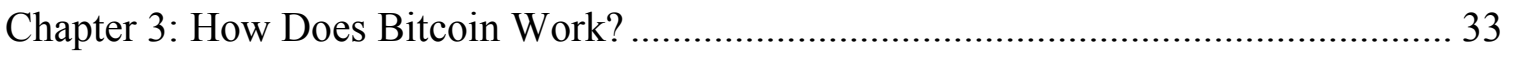

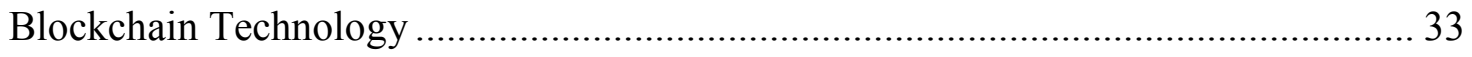

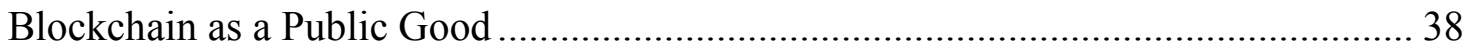

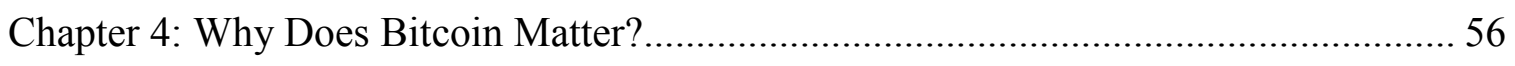

Bitcoin as a Social Movement: A Comparative Analysis........................................ 56

How Bitcoin and Blockchain are "Shaking the System" ......................................... 77

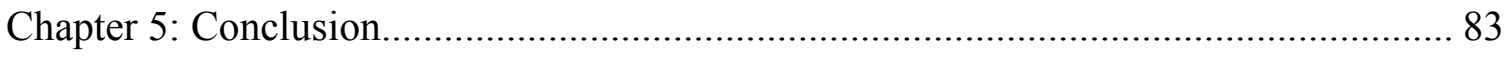

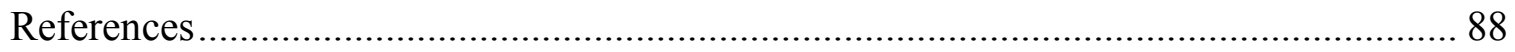




\section{List of Tables}

Table 1. Minimum Annual Number of Unique Bitcoin Miners, 2009-2019 ...................52

Table 2. Summary of Findings: The Cypherpunks and Bitcoin as Contentious Politics...75 


\section{List of Figures}

Figure 1. Average Number of Daily Bitcoin Transactions \& Price..................................16

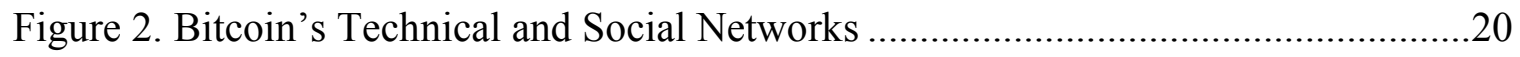

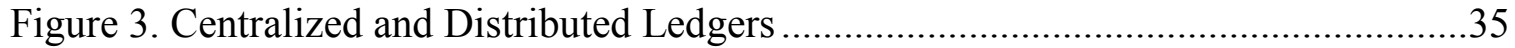

Figure 4. Would-Be Bitcoin Miner Game Payoff Matrix, Threshold Believed Not Met..50

Figure 5. Would-Be Bitcoin Miner Game Payoff Matrix, Threshold Believed Met.........51

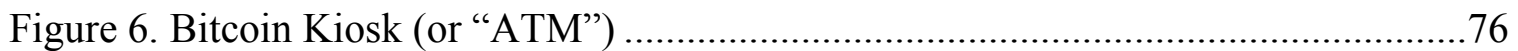


Preface

On October 23, 2019, Facebook CEO Mark Zuckerberg testified before the United States House of Representatives Financial Services Committee. A year and a half earlier, Zuckerberg sat before a joint session of the US Senate commerce and judiciary committees. Then, he was responding to concerns over Facebook's alleged violations of user privacy and its role in the 2016 US elections. This time, Congress's inquiry would be more concrete and more proactive. During the hearing entitled "An Examination of Facebook and Its Impact on the Financial Services and Housing Sectors," Zuckerberg defended his company's "Libra" project, a blockchain-based cryptocurrency revealed on June 18, 2019. Cryptocurrencies are digital payment systems that use cryptography to operate securely without a third-party intermediary. A blockchain is a publicly accessible digital ledger of transactions that can be implemented with varying degrees of privacy. The change of venue and speed with which the Facebook hearing took place are telling: after having been probed for social media's role in political campaigns, what does Facebook have to do with financial services? Libra is not yet operational, but it aspires to be "a simple global currency and financial infrastructure that empowers billions of people" (Libra Association 2019).

Three months before Zuckerberg's Libra testimony, Facebook's David Marcus gave the Financial Services Committee a primer on cryptocurrency. The Committee $(2019,2)$ found that "Facebook's plans have serious implications for investors, consumers, data privacy, cybersecurity, systemic risks, monetary policy, and national 
security." The Independent Community Bankers of America testified that "the proposed creation of Libra, if allowed to proceed, would be a significant and irreversible development that would alter the global financial landscape.” Federal Reserve Board Chairman Jerome Powell commented that "Libra raises many serious concerns regarding privacy, money laundering, consumer protection, and financial security," while the Fed's Governor Lael Brainard added, "The potential for risks and spillovers could be amplified by potential ambiguity surrounding the ability of official authorities to provide oversight and backstop liquidity, and to collaborate across borders." In the time between Marcus's and Zuckerberg's testimony, France's finance minister Bruno Le Maire (2019) described Libra as "asking states to share their monetary sovereignty with private companies."

Policymakers' views of the technology upon which Libra is based are, however, complicated. China has banned cryptocurrency exchanges and trading platforms (Rapoza 2017), prohibited financial institutions from engaging in Bitcoin transactions (Bloomberg News 2013), and taken steps to suppress Bitcoin mining (Chen and Ren 2018). Still, China and other states, including Sweden, Dubai, and Venezuela, are developing their own, state-issued digital currencies (the digitized renminbi, e-krona, emCash, and Petro). Other countries are more reluctant to employ the technology, attempting to support private sector innovation without ceding too much discretion. While legal to use in the US, there is little interagency agreement as to how to treat cryptocurrency. ${ }^{1}$ The state of

\footnotetext{
${ }^{1}$ For example, the Office of the Comptroller of the Currency is exploring national bank charters for financial technology firms such as those developing cryptocurrencies, the Securities and Exchange Commission is formalizing whether or not specific cryptocurrency investments constitute securities, and New York state started licensing digital currency companies with "BitLicense" in 2015.
} 
Ohio now accepts business tax payments in bitcoin $^{2}$ as it angles to become a blockchain technology hub (Ma 2019), whereas many observers consider New York’s “Bitlicense” regulatory regime the nation's most stifling (Bryanov 2019). Ultimately, as this thesis intends to show, the impulses and tensions behind electronic cash run far deeper than just taxation and regulation. They are acutely bound up in money and what one can learn from people's use of it.

${ }^{2}$ According to convention, the word "Bitcoin" is capitalized when discussing Bitcoin as a network, system, or technology; bitcoin is not capitalized when discussing the unit of cryptocurrency. 


\section{Chapter 1: Introduction}

\section{"[...] anything that is using distributed ledger technology [...] is clearly shaking the} system."

- Christine Lagarde, as Managing Director of the IMF (in Schulze 2019)

Every ten minutes, the Bitcoin network deploys more processing power than all of Google's servers combined. It consumes enough electricity to power the Philippines, and if its market capitalization were equivalent to GDP, it would rank in the top third of all countries. The most reliable estimates indicate there were at least 35 million cryptocurrency users worldwide as of December 2018, for whom Bitcoin is dominant (Rauchs et al. 2018). ${ }^{3}$ Bitcoin network nodes were active in 99 countries as of May 2020 (Yeow 2020). In other words, Bitcoin is transnational phenomenon of national proportions, built entirely around an untraceable electronic cash system. Yet political scientists have had little to say about Bitcoin. In fact, in a search of leading political science and international relations journals, the word "Bitcoin" appears in zero article titles. $^{4}$

To situate Bitcoin in the international relations literature, I argue that Bitcoin is a form of contentious politics uniquely suited to the twenty-first century: it is a global electronic cash system built for pseudonymous peer-to-peer exchange. As such, Bitcoin

\footnotetext{
${ }^{3}$ The precise number of Bitcoin users is difficult to estimate given user pseudonymity.

${ }^{4}$ Author's search conducted May 2, 2020 from among the following journals: American Journal of Political Science, American Political Science Review, Comparative Political Studies, The Journal of Politics, British Journal of Political Science, International Organization, Third World Quarterly, Journal of Democracy, International Studies Quarterly, and World Politics.
} 
severs the connection between identity and electronic money, subverting states' enforcement capabilities by disrupting their ability to monitor financial flows. The implications for the state in an increasingly digital, global environment are significant. Bitcoin and related blockchain technologies facilitate illicit financial flows, such as those involved in crime and terrorism, making it difficult for enforcement agencies to track such flows. Furthermore, if the share of overall economic activity conducted via Bitcoin and related technologies makes inroads compared with conventional currencies, then states will need to adjust in order to monitor, regulate, and tax such economic activity. In developing this argument, I organize this thesis as a series of three questions, each answered for an audience interested in Bitcoin and blockchain's implications for international relations and international political economy. In Chapter 2, "What Is Bitcoin?" I provide a technical description of Bitcoin, briefly discuss the origins of electronic cash, and evaluate Bitcoin as an actor in global affairs. I find that Bitcoin's success as a transnational cyber actor is mixed: it has subverted states' authority only for a portion of its users. Still, given its ability to shield users from monitoring and enforcement, Bitcoin should not be taken lightly. In Chapter 3, "How Does Bitcoin Work?" I present an introduction to blockchain technology and conceptualize blockchain as a public good. In so doing, I conceive of Bitcoin as a public goods provider and apply game theory to model how the network coordinates the voluntary provision of resources from among its users. In Chapter 4, "Why Does Bitcoin Matter?" I evaluate Bitcoin as a social movement, comparing it with the Cypherpunks as a predecessor of direct cyber action. I follow the comparative analysis by considering exactly how, in the words of Christine Lagarde, Bitcoin and blockchain are "shaking the system." I conclude the thesis 
by considering potential research avenues into Bitcoin as a form of contentious politics and by outlining how lessons learned through Bitcoin may apply to its technological descendants, including blockchain technology, central bank digital currencies, and Facebook Libra.

There is much about Bitcoin to find both fascinating and perplexing. Indeed, simply explaining what Bitcoin is, is fascinating and perplexing. Beyond that, the story of Bitcoin offers a cutting-edge account of twenty-first century global affairs, state-society relations, the power of technology, innovative attempts to solve collective action problems, and how politics unfolds in cyberspace. Some are convinced that Bitcoin is a revolutionary technology. Others, just a passing fad. In crafting this thesis, I submit that Bitcoin, in one form or another, it is here to stay and is worth political scientists taking note. 


\section{Chapter 2: What Is Bitcoin?}

Chapter 2 begins with a technical description of Bitcoin, followed by a brief story of its origin. The majority of this chapter is devoted to evaluating Bitcoin as an actor in global affairs. I argue that Bitcoin has faced a paradox to date: its political purpose has undermined its economic imperatives while its economic imperatives have undermined its political purpose. In short, Bitcoin's political purpose is to subvert the state through untraceable electronic cash. As a form of cash, its user base must grow to engage positive network externalities. However, as the Bitcoin network developed, profit-seeking service providers have become responsible for an increasing share of Bitcoin user growth. In practice, such service providers violate the mechanism by which Bitcoin subverts state

authority, pseudonymity. This is not to say that Bitcoin has been wholly unsuccessful as a subversive tool. In fact, as the analysis shows, Bitcoin continues to serve the subversive interests of those taking the care to preserve their pseudonymity.

\section{Technical Description of Bitcoin}

In an early technical study of Bitcoin, Joseph Bonneau et al. (2015, 105-109) describes Bitcoin as comprising three technical components: transactions, a consensus protocol, and a communications network. A Bitcoin transaction is a message containing a set of outputs and inputs, moving from one (or several) Bitcoin addresses to other address(es). Each output represents a sum of bitcoin, often denoted by the symbol $\square$ or the abbreviation BTC. The set of inputs comprises outputs from previous transactions, much like physical bills or coins pass from one party to another and another. Transacting 
in bitcoin relies on modern cryptography, in that a user must know what is called a "private key" to digitally sign (and thus execute) a transaction. Knowledge of a private key associated with an address (or "public key") imparts control over the bitcoin linked to that address.

Among several other functions, the consensus protocol, "Bitcoin's core innovation and perhaps the most crucial ingredient to its success" (Bonneau et al. 2015, 106), ensures bitcoins are not fraudulently reproduced (known as "double spending"). As digital artefacts, bitcoin outputs run the risk of being cheaply and easily duplicated, akin to counterfeiting physical money. The consensus protocol prevents such duplication by publishing valid transactions to the network, to be seen by all and against which future transactions are checked. To validate transactions, specialized nodes in the network, called "miners," devote computing power in exchange for the possibility of receiving newly minted bitcoin. The more computing power a miner devotes to the network, the greater the probability they have to receive the next minted bitcoin, setting up a competitive mining environment. ${ }^{5}$ Miners package hundreds of validated transactions into a set called a "block," and the first miner to successfully add a block to the network by solving a complex cryptographic puzzle wins the "block reward" and any transaction fees attached to the transactions therein. The newly mined block is added to the set of previously mined blocks, containing a snippet of code that links it immutably to the set of prior blocks. The result of this process is known as the "blockchain."

\footnotetext{
${ }^{5}$ The competitive nature of mining is designed to limit the possibility that any one miner would secure over $50 \%$ of the network's computing power, enabling the miner to validate fraudulent transactions or single handedly change the network protocol. This $50 \%$ threshold is a critical value in the mining network.
} 
Bitcoin's communications network enables rapid transmission among nodes by connecting each node to a random sample of several other nearby nodes. ${ }^{6}$ Bitcoin's communications protocol enables the near-instantaneous synchronization of the transaction ledger among all nodes active on the network, meaning that all nodes simultaneously share the same view of transactions, what has become known as a "distributed ledger." Every transaction is broadcast to the network, whereby it is validated (primarily to ensure inputs used had not been previously spent) and becomes written to the ledger.

Together, these three technical components make the Bitcoin network operational. It is, perhaps, a network in pure form. Bitcoin has no institutional sponsor (there is no "Bitcoin corporation" or sponsoring organization from which the project was launched): it is merely a collection of computers sharing a set of protocols.

\section{The Origins of Electronic Cash}

At the click of a mouse, a mysterious figure known by the pseudonym "Satoshi Nakamoto" introduced Bitcoin. That was just over eleven years ago, during the depths of the Great Recession. On an otherwise unremarkable Friday evening (UTC) of October 31, 2008, Nakamoto (2008a) posted to The Cryptography Mailing List for the first time. The email's subject line, "Bitcoin P2P e-cash paper," and its one-line introduction, "I've been working on a new electronic cash system that's fully peer-to-peer, with no trusted third party," seem understated in hindsight. Electronic cash refers to a form of digital

\footnotetext{
${ }^{6}$ When a node transmits a message (such as a transaction or mined block), the message rapidly propagates among all nodes by beginning with its direct neighbors, each of which in turn relays the message to their direct neighbors, cascading the message throughout the network.
} 
currency that, like cash, may be used without necessarily identifying its user. Since the emergence of online interaction, particularly online bulletin board systems (BBSes) in the 1980s, developing electronic cash had become an interest of many concerned with online privacy. Until Bitcoin, no attempt at electronic cash lasted long. Reflecting on the state of electronic cash, Nakamoto (2009) writes:

A lot of people automatically dismiss e-currency as a lost cause because of all the companies that failed since the 1990's. I hope it's obvious it was only the centrally controlled nature of those systems that doomed them. I think this is the first time we're trying a decentralized, non-trust-based system.

Whereas previous models of electronic cash — including DigiCash, e-gold, and bit goldrelied in some part on a trusted third party to function, Bitcoin did not. Nakamoto's (2008b, 1) white paper, "Bitcoin: A Peer-to-Peer Electronic Cash System," outlines the technical features of Bitcoin, describing it as "a purely peer-to-peer version of electronic cash [that] would allow online payments to be sent directly from one party to another without going through a financial institution." Uploading the white paper to the newly created bitcoin.org, Nakamoto (2008c) identifies Bitcoin’s three “Main properties:

-Double-spending is prevented with a peer-to-peer network.

-No mint or other trusted parties.

-Participants can be anonymous."

Two months later, Bitcoin went live when Nakamoto "mined" its "genesis block," dated January 3, 2009. Bitcoin was born from obscurity, introducing several novel concepts and solving once intractable technological issues. Next, it would need to grow.

Its first user joined on January 10, 2009, when Hal Finney, a developer for PGP Corporation and close collaborator of Nakamoto's, downloaded and installed the Bitcoin code, connecting to the network. "Apart from Hal," Nathaniel Popper $(2015,5)$ describes the moment Bitcoin went live, "there were only two other computers on the network and 
both of those were from a single IP address, presumably Satoshi's, tied to an Internet provider in California." Finney set his machine to work "mining"-what Nakamoto called the proof-of-work process that generated new bitcoin—and left for dinner with his family. By the time he returned, his computer had mined 50 bitcoins, posted to his personal Bitcoin address and recorded publicly on the Bitcoin ledger. ${ }^{7}$ Since then, Bitcoin has attracted millions of users worldwide.

\section{Bitcoin as an Actor in Global Affairs}

In this section, I argue that Bitcoin faces a paradox: its political purpose undermines its economic imperatives while its economic imperatives undermine its political purpose. To evaluate Bitcoin's political effectiveness, this section defines Bitcoin as a transnational cyber actor and applies the network-as-actor model of network analysis. I find that Bitcoin's economic features have largely benefited at the expense of its political purpose.

The relationship between the Internet and politics has come into sharp focus of late. Taken as a venue of political contestation, the Internet's uses and effects are subject to wide ranging debate (Coleman and Freelon 2015, 3-8). Whether or not the Internet has accomplished its original design purpose - to make the world, or at least the US, a safer, more connected place (Leiner et al. 1997) — is debatable. It was only in 2008 when a candidate for US President first devoted substantial campaign resources to their Internet media, and only two election cycles later, allegations surfaced that the Kremlin directed

\footnotetext{
${ }^{7}$ As of May 14, 2020, Finney's first 50 bitcoins would be worth approximately $\$ 483,000$. One can view the record of this particular transaction by searching online for block 78 of Bitcoin's blockchain.
} 
election meddling in the 2016 US presidential race. Social media propelled the Arab Spring, but it may also enable authorities to target and suppress opposition (Dewey et al. 2012, 28-30). Ongoing protests in Hong Kong are covered in detail to a world of Internet users, but the details vary depending on what side of the Great Firewall they land (Mahtani, Shih, and Liang 2019). Indeed, politics has shaped the Internet perhaps as much as the Internet has shaped politics.

As an offshoot of the Internet, Bitcoin's political purpose is to subvert the state through untraceable electronic cash. As a form of cash, its user base must grow to engage positive network externalities. However, as the Bitcoin network developed, profit-seeking service providers have become responsible for an increasing share of Bitcoin user growth. In practice, such service providers violate the mechanism by which Bitcoin subverts state authority, pseudonymity.

Transnational Cyber Activism: The Case of Bitcoin

As the Internet gains significance in global affairs, examining transnational cyber activism becomes increasingly important. Cyber activism takes advantage of information and communications technologies (ICT) such as the Internet to achieve political objectives (Carty 2015, 35). Cyber activism uses ICT to identify, articulate, and promote common goals; to organize and mobilize activists; and to monitor and enforce behaviors. Using the Internet also offers transnational cyber activists varying degrees of protection from third-party surveillance.

Cyber activists' political objectives may also threaten regime stability. Globally organized via the Internet, transnational cyber actors assume a network form of 
organization, wherein interdependent actors are connected through social relations. Political scientists typically study such networks through two approaches: network-asstructure and network-as-actor (Kahler 2009). As structures, social networks embody "relational structures $[\ldots]$ or emergent attributes [...] that systematically influence the actions of its nodes (members) and produce identifiable outcomes" (4). As actors, transnational social networks are "forms of coordinated or collective action aimed at changing international outcomes and national policies" (5).

Miles Kahler's (104) framework for analyzing a network-as-actor inspires the following questions:

- To what degree can transnational cyber activists be defined as networks (as opposed to other forms of organization such as markets, hierarchies, or movements)?

- Is the network an effective form of organization for achieving cyber activism?

- How does transnational cyber activism affect network structure and performance? I begin by examining the Bitcoin network as a transnational cyber actor. Bitcoin is a global, "peer-to-peer" electronic payment system that operates as a network of computers connected over the Internet. Bitcoin's fundamental purpose is distinctly political: creating electronic cash that is not subject to state authority. Bitcoin attempts to do so by subverting the state's ability to monitor individual behavior. Specifically, Bitcoin enables users to conduct exchange with one another pseudonymously, eroding states' ability to identify users and thus thwarting enforcement action. Operating outside the international monetary system further minimizes state influence over Bitcoin. It is important to note that Bitcoin operates without any formal authority other than its code, 
the Bitcoin communications protocol. This is why Bitcoin's creator describes it as "purely peer-to-peer." It is also perhaps the most thoroughgoing application of Lawrence Lessig's (1999) pithy remark on governance in cyberspace, "code is law."

Over time, Bitcoin has become increasingly significant in international affairs. It is a global phenomenon, with millions of users worldwide. Bitcoin's user base has grown exponentially since its 2009 release, with a majority having first purchased Bitcoin after 2016. Policy makers are sending increasingly critical signals about Bitcoin and its underlying technology. In April 2019, International Monetary Fund Managing Director Christine Lagarde summed up the sentiment: "I think the role of the disruptors and anything that is using distributed ledger technology, whether you call it crypto, assets, currencies, or whatever [...] is clearly shaking the system" (in Schulze 2019).

The literature on Bitcoin in international affairs is nevertheless scant. This analysis seeks to offset the lack of scholarship in the field by relying primarily on scholarship outside the field as well as carefully vetted journalism. Additionally, the nature of the dependent variable under examination in this section, the informal cyber economy, is subject to measurement challenges: it is easier to observe the formal cyber economy than the informal. As such, in examining the effects of Bitcoin's network form of organization on its political success, this section is a descriptive case study. It draws heavily on Kahler's (2009, 103-124) method of analysis in "Collective Action and Clandestine Networks: The Case of Al Qaeda." 


\section{Bitcoin's Multiple Networks}

This section focuses on the emergence of multiple organizational authorities within Bitcoin's social network. Atop Bitcoin's technical network, a complex and multidimensional ecosystem has formed. Much like various applications, platforms, and practices have grown atop the Internet, new services and practices are growing atop Bitcoin's technical infrastructure. Much of the growth of this superstructure is designed to facilitate consumer and investor participation in the Bitcoin network. While it is technically possible for anyone with Internet access and a computer to join the Bitcoin network by running a node, doing so requires a degree of coding knowledge and disposable time. As a result, a cottage industry of service providers has emerged to make Bitcoin interfaces more user-friendly and transacting in bitcoin convenient. Now, anyone who can connect to the Internet or send and receive SMS messages can use bitcoin.

Michel Rauchs et al. $(2018,19)$ classifies this Bitcoin service industry into several segments: mining, storage, payments, and exchange. During 2009-2010, mining bitcoin could be accomplished by individuals with widely available computers. As mining became more competitive, it required more computing power to succeed. In late 2010 , the first "mining pool" emerged, "Slushpool," which aggregated the computing power of multiple individuals' units into a single miner, making it able to compete with even the most powerful computers involved. Slushpool then distributed mining rewards among its members according to how much computing power each contributed, developing the pool model that now dominates mining in Bitcoin. By offering convenient ways to secure their bitcoin and engage in transactions, companies in the storage, payments, and exchange segments facilitate Bitcoin use by consumers and investors. Payment service providers, 
for example, offer retailers a way to accept bitcoin as payment for goods and services. Exchanges make it easy to purchase bitcoin with a credit card or bank account denominated in local currency. Bitcoin ATMs facilitate purchasing bitcoin with cash. While this wider ecosystem provides access to a much larger group of users than otherwise may have participated, because such services re-introduce third parties to the network, their prevalence departs from Bitcoin's fundamental basis as a purely peer-topeer medium.

Bitcoin's multidimensional ecosystem also reflects ideological diversity within the Bitcoin community. Ideological debate with respect to Bitcoin centers largely on its function as electronic cash. While the most enduring, Bitcoin was not the first implementation of electronic cash. The impulse to create electronic cash originally surfaced in the cryptographic research community. Its leading proponent during the 1980s, David Chaum (in Levy 2001, 215) argued that electronic cash was necessary to offset technological trends toward an "enormous surveillance potential [that would] leave individual's lives vulnerable to an unprecedented concentration of scrutiny and authority." Privacy was the central concern. Chaum's attempts to implement electronic cash — the most recognizable having been "DigiCash"-were instrumental, but ultimately unsuccessful contributions. In the 1990s, a loosely affiliated group of hackers known as the Cypherpunks committed to develop electronic cash as a bulwark against what they perceived as a growing surveillance state. Well-respected among Cypherpunks, Tim May and Eric Hughes imbued the Cypherpunk worldview with an anarcho-capitalist orientation, promoting an extreme free-market posture (Ludlow 2001). Not all Cypherpunks shared the worldview, however, having noted the growth of corporate 
power in the marketplace, and developers such as Nick Szabo called for ideological temperance.

Bitcoin ultimately addressed the privacy concern. Unless otherwise revealed, Bitcoin addresses are not necessarily associated with identities, making pseudonymous ownership of bitcoin possible. Bitcoin's creator, Satoshi Nakamoto, clearly identified with the cryptographic community, having built Bitcoin on modern cryptographic methods, referencing leading cryptographers in their work, and originally introducing Bitcoin to an online cryptography community. Given Nakamoto's interest in designing an intermediary-free transaction system, their reference of known Cypherpunks, and a cryptic note appended to the very first bitcoin, ${ }^{8}$ some associate Nakamoto with the Cypherpunks. However, because Nakamoto had little to say about ideological matters and has been unheard from since 2011, it is impossible to further elaborate on the original ideological intent behind Bitcoin. That has not stopped some from trying, most notably commentators including Saifedean Ammous (2018) who allege Bitcoin was established on Austrian School economic principles because of Bitcoin's predictable, automated deflationary money supply. There are also those whose ideological bearings are less explicit and are primarily concerned with matters of utility: Bitcoin attracts speculators, investors, and entrepreneurs who may be characterized as pragmatic in their approach to Bitcoin.

\footnotetext{
${ }^{8}$ On the first bitcoin block ever mined (the "genesis" block), Nakamoto inscribed the headline "Chancellor on brink of second bailout for banks," from the January 3, 2009 edition of The Times as proof the block had not been mined any earlier than that date. Some observers read it as an ideological statement, given it was made at the height of the Great Recession, and insinuates a critique of monetary authorities and large banks.
} 


\section{The Bitcoin Social Network's Three Stages of Development}

Bitcoin's social network structure has undergone three stages of development since its inception: formation, rapid growth, and uncertainty. Price and transaction patterns, evolving uses, and differing public perceptions characterize each stage. Figure 1 portrays the network's growth over time by transaction volume and price. The orange points reflect the average number of daily transactions (left axis, logarithmic scale), indicating changes in network size and scale over time. The blue line reflects bitcoin's market price in US dollars (right axis, logarithmic scale). Since the supply of bitcoin is relatively stable, its price should be a reliable measure of demand for bitcoin, and consequently a proxy for relative user interest over time.

The first stage of Bitcoin development, formation, occurred from October 2008 until mid-2010. Low, insular activity and a series of firsts characterize the formation stage. After releasing the Bitcoin software on January 3rd, 2009, Nakamoto created an online Bitcoin forum to publicize and discuss Bitcoin. Members trickled in. While there was no market for bitcoin trading, a respected Bitcoin forum member known as NewLibertyStandard first priced bitcoin at 1,309.03 to $\$ 1.00$ in October 2009. Within months, the first bitcoin exchange, "Bitcoin Market" launched, enabling users to trade bitcoin. In May 2010, computer programmer Laszlo Hanyecz made the first purchase using bitcoin by sending 10,000 bitcoins to a stranger in exchange for pizza delivered in Jacksonville, Florida (Popper 2015, 42-44). At the time, one bitcoin was not even worth a penny. 
Figure 1. Average Number of Daily Bitcoin Transactions \& Price

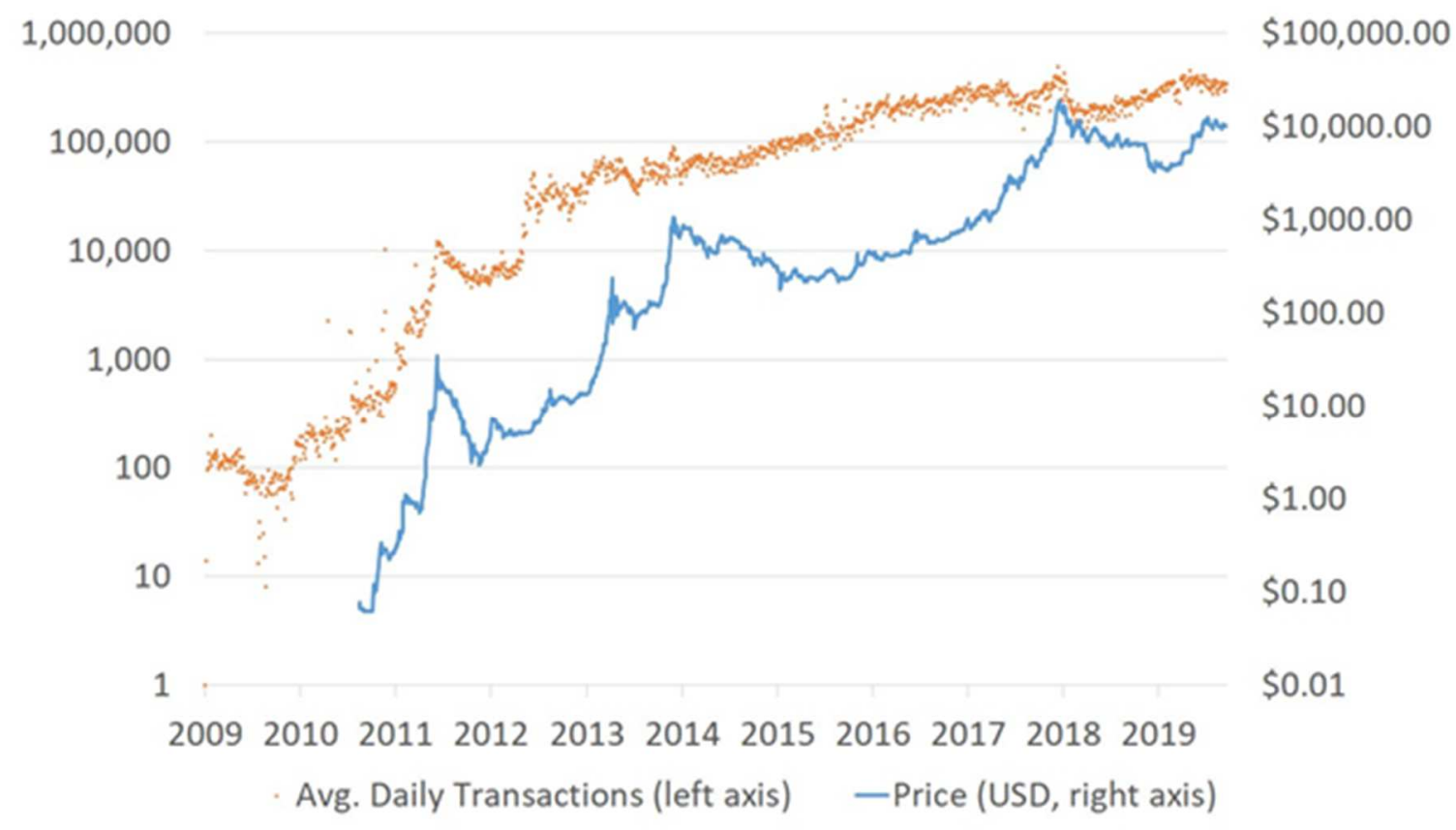

Source: Blockchain.com (2019).

In the latter half of 2010, Bitcoin progressed beyond formation and into a rapid growth stage. What would become Bitcoin's largest exchange, Mt. Gox, launched in July 2010, and Bitcoin's first mining pool, Slushpool, started operating in November 2010. Mt. Gox was the first exchange to make purchasing bitcoin with conventional currency possible, by accepting PayPal payments (in violation of PayPal's terms) in exchange for bitcoin (Popper 2015, 52). At the same time, the difficulty of mining for bitcoin increased dramatically as competition grew. Increased mining difficulty led to the emergence of mining pools and made purchasing bitcoin through exchanges the most reliable method of obtaining bitcoin.

The following year, popular media stories about Bitcoin proliferated, with features in TIME, Gawker, and Wired spotlighting the technology and drawing attention 
to its rising use in illicit activities. Adding to the public image of Bitcoin's use in illicit activities, in October 2013, US authorities confiscated the anonymous online marketplace “Silk Road." Silk Road had operated since 2011, providing a platform for merchants to sell drugs, weapons, and illegal services for an estimated total annual volume of $\$ 14.4$ million, entirely in bitcoin (Christin 2013, 1). After a major hack led to losses approaching $7 \%$ of all bitcoin in circulation, Mt. Gox filed for bankruptcy protection in February 2014 (Takemoto and Knight 2014). The Financial Action Task Force (FATF), an intergovernmental anti-money laundering and anti-terrorism financing organization, released a critical report on virtual currencies including Bitcoin in June 2014. The Silk Road and Mt. Gox failures, as well as increasing media and governmental scrutiny did little to restrain Bitcoin's growth. By then, a significant group of users had been convicted of Bitcoin's long-term prospects and publicly devoted themselves to holding bitcoin at any price. They may have been justified because since June 2014, bitcoin's price increased from about $\$ 615$ to nearly $\$ 10,000$.

After a period of rapid appreciation during 2017, Bitcoin reached a stage of uncertainty. Prices have nearly halved from their December 2017 peak and volumes have wildly oscillated between 150,000 and 400,000 transactions per day. Consumers and investors appear to have tempered their expectations, while some influential corporations have embraced blockchain. Multinational financial firm J.P. Morgan partnered with software giant Microsoft in 2018 to establish "Quorum," a blockchain platform built for large corporations. Nearly 90 corporations, government agencies, and academic institutions have joined to support the Blockchain Research Institute, founded in 2017 to 
research blockchain applications in a wide range of sectors. Facebook announced its own cryptocurrency project, "Libra,” in June 2019.

Authorities have also turned greater attention toward Bitcoin and the thousands of "altcoins" (cryptocurrencies modelled after Bitcoin) it has spawned. In September 2017, the Bank for International Settlements, an intergovernmental monetary and financial stability watchdog, published a report on the potential of central bank cryptocurrencies to compete with the likes of Bitcoin (Bech and Garratt 2017). Speculation that China is developing its own central bank cryptocurrency grew more palpable as this thesis was written (Orcutt 2019). The Federal Reserve Bank of Saint Louis, a key repository of economic data, added cryptocurrency data series to their reports in 2018. And in June 2019, the FATF released stringent new guidelines targeting cryptocurrency companies (FATF 2019, 55).

\section{Bitcoin's Social Network Map}

As Bitcoin grew, various types of actors emerged to join the network, including both individuals and organizations. Individuals may use bitcoin either directly or through service providing organizations. Each organization (such as an exchange, a merchant, or a mining pool) acts as a single, service providing node in the network. Such organizations comprise respective sets of member units, either individuals or other organizations. Links connecting nodes with one another, as well as links connecting nodes with actors outside the network, are fundamentally communications links conveying the flow of information. Figure 2 presents a stylized network map conveying node characteristics and link dynamics with respect to Bitcoin's political purpose. Recall that Bitcoin relies on 
pseudonymity to disrupt states' ability to monitor individual behavior and target enforcement. The network map draws attention to features that complicate pseudonymity, making bitcoin use subject to state intervention. Each node acts on behalf of its user(s) from the social network. A single node, such as an exchange node, may have multiple users, and a single user may operate one or more nodes. Links between users and nodes represent flows of personally identifying information: solid lines denote identity disclosure (the arrow pointing to the recipient) and dashed lines denote the use of pseudonyms. Shaded nodes possess their users' identities; unshaded nodes do not. Links between nodes represent communication, including possible transactions.

What follows from a glance at the stylized network map is the need to investigate network structure with respect to the relationships within the network and relationships between network nodes and the outside world. It is clear from the outset that where individuals are vulnerable to monitoring, they are connected to the network through service providers. When selling bitcoin for fiat currency, for example, exchanges typically require users to identify themselves to comply with international Know Your Customer (KYC) and Anti-Money Laundering and Combating the Financing of Terrorism (AML/CFT) controls. In turn, should a state wish to identify exchange users, it may turn to the exchange operator. By contrast, users who pseudonymously transact on the technical network withhold their identities, making them improbable subjects of enforcement. 
Figure 2. Bitcoin's Technical and Social Networks

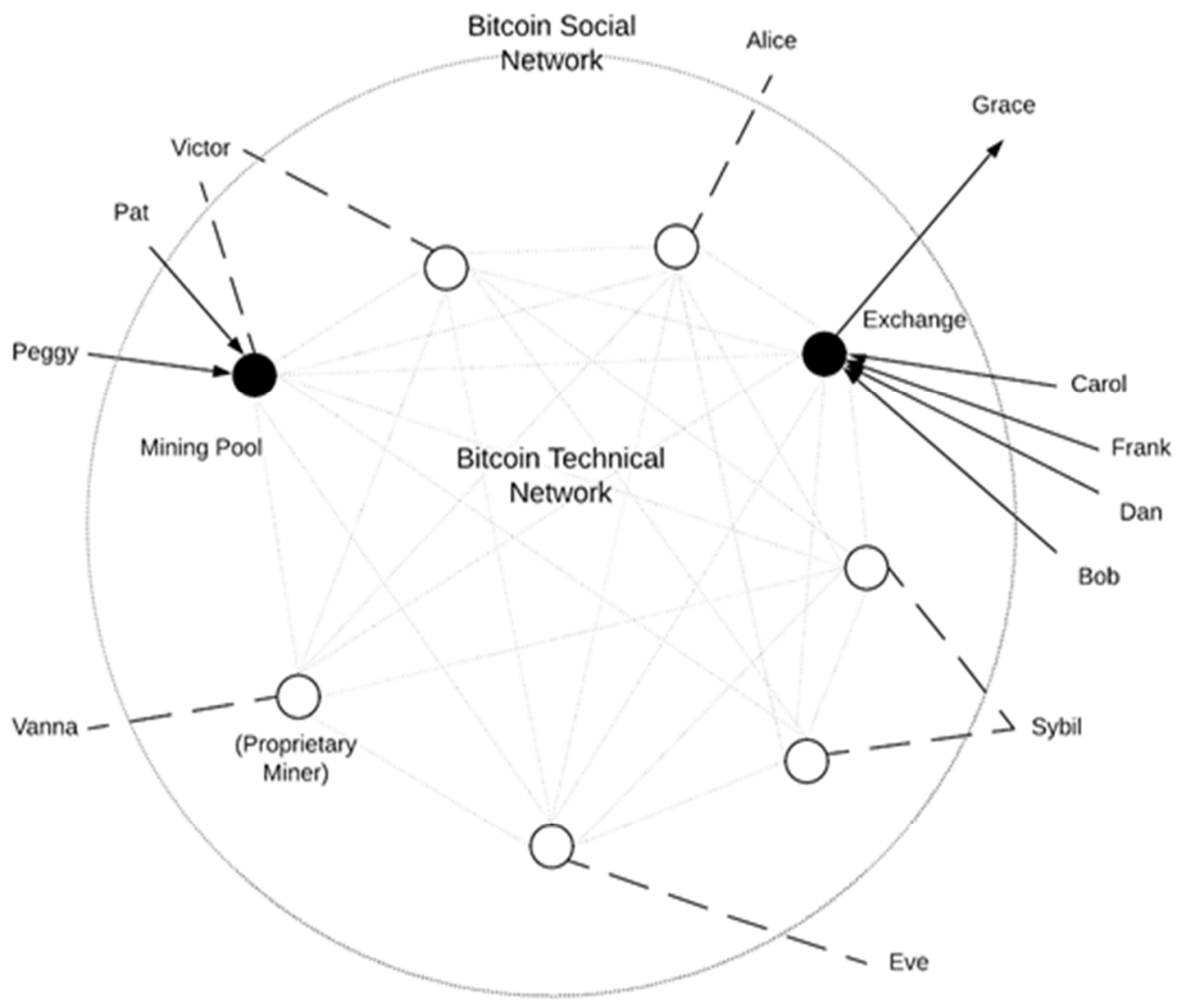

Link:
Technical (Communication)
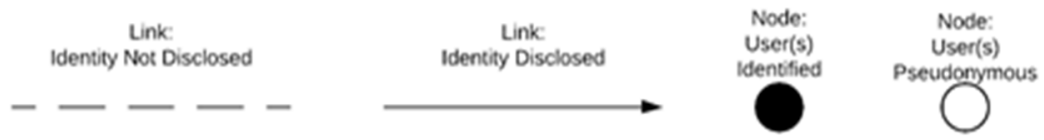

Service nodes introduce hierarchy to the network, in the process making their members vulnerable to state intervention. A corollary vulnerability is that as the importance of service nodes increases in relation to individual nodes, the network's resilience diminishes because it loses its distributed structure. Given the different characteristics of individual user nodes and service provider nodes, the most potential for political success through the Bitcoin network-as-actor is through increasing its proportion 
of individual user nodes. Increasing individual user node proportion is necessary for three reasons. First, it is how Bitcoin increases the size of its informal economy. Second, as Michael Katz and Carl Shapiro $(1994,94)$ point out discussing positive network externalities, "the value of membership to one user is positively affected when another user joins and enlarges the network." Third, the greater the proportion and activity of direct participation, the more distributed — and thus resilient— the network becomes.

\section{Collective Action and Bitcoin}

Following Kahler's $(2009,109)$ model of collective action, this section examines three key features of the Bitcoin network-as-actor: 1) social networks and embeddedness, 2) network links, and 3) network structure. To begin, the external social networks to which Bitcoin users belong, such as interest groups or criminal networks, can have collective action consequences. Such users provide outsiders on-ramps to the network, whether adversarial, friendly, or indifferent. In a similar way, the degree in which Bitcoin is embedded within wider patterns of interaction can expose the network to threats and opportunities.

Links "that represent the flow of resources or information" (Kahler 2009, 110) in the Bitcoin network may also have consequences for collective action. Networks often distribute resources as a means to stimulate collective action. Resources may be material such as cash or non-material such as influence or status. Positive network externalities increase the value of resources to users: the more users, the more valuable such resources become. Information can also foster collective action "through the quality of information, information processing capabilities within networks, and the diffusion of information that 
illuminates behavioral interdependence" (110). Indeed, Bitcoin is built on the quality of information retained by its immutable, validated, and public blockchain. Bitcoin is also lauded for its information processing potential: hence, the corporate hype around blockchain. Finally, information diffusion may support collective action by signaling behavioral norms to its users.

Network structure may affect collective action through the patterns of connection among network members. Networks characterized by high degrees of centralizationwhen subsets of network members are strongly connected with particular members but weakly connected to the wider network - are challenged to take collective action beyond the subset to the level of the network. While such strongly connected subsets may be successful collective actors in and of themselves, their ties do not typically spread among the entire network. To raise collective action to the level of the network, weaklyconnected members (with links to members of different subsets) may be critical to achieving collective action across the network.

\section{Agenda Setting in a Distributed Network}

Bitcoin began as a white paper posted to a rather obscure cryptography mailing list in 2008. Its purpose was "allowing any two willing parties to transact directly with each other [in cyberspace] without the need for a trusted third party" (Nakamoto 2008b, 1). In other words, Bitcoin attempts to create electronic cash that is not subject to state authority. In today's monetary system, the state is the ultimate "trusted third party," compelled to "preserv[e] market confidence in the value and usability of the nation's money" (Cohen 2008, 465). The state also oversees and regulates a key set of trusted 
third parties, financial intermediaries such as banks and payment processors. At the outset, Bitcoin's political success depended on users joining the network to increase positive network externalities and users behaving pseudonymously to shield their actions from the state.

Bitcoin's agenda became contested during its rapid growth stage. Nakamoto abruptly disappeared in 2011, creating a vacuum in informal, but respected agenda setting authority. Concurrently, the entrance of Bitcoin service providers such as exchanges, mining pools, and merchants introduced profit-seeking enterprises to the network. Czech Republic based Slushpool was the first Bitcoin mining pool, a service that allows subscribers to contribute computing power in return for a share of proceeds from Bitcoin mining. Slushpool is now one of dozens of profit-seeking mining pools, most based in China. An early Bitcoin exchange founded in 2010, Mt. Gox processed about $80 \%$ of transactions using bitcoin at the height of Mt. Gox's business (Abrams and Popper 2014). A series of hacks between 2011 and 2014 bankrupted Mt. Gox. For Slushpool and Mt. Gox, the pecuniary motive supplanted political principle as their leaders spoke more about bitcoin's economic features than pseudonymity. An illicit market, "Silk Road," operated from 2011 through 2013, becoming the dominant bitcoin merchant at the time. US authorities ultimately shut the operation down in 2013. Silk Road's conduct and demise may be one of the most significant sources of the public's lingering perception of Bitcoin as an instrument of crime (Foley, Karlsen, and Putniņ̌̌ 2019). Numerous Bitcoin spokespersons emerged with varied, sometimes fraudulent motives and get-rich-quick stories (Popper 2015), further diluting Bitcoin's agenda. Finally, increased regulatory attention appears to have inspired other influential actors, such as the advocacy 
organization Coincenter and the exchange Coinbase, to embrace the less politically threatening features of Bitcoin. While uncertainty may characterize Bitcoin's current phase, the agenda of its most influential actors appears to have settled on Bitcoin as money or investment, rather than as a political experiment.

\section{Social Networks and Embeddedness}

Bitcoin's political purpose likely benefited during its formation stage from two social networks that had existed before Bitcoin: the cryptography mailing list and the Cypherpunks mailing list. Satoshi Nakamoto had been a contributor to the cryptography mailing list, while Hal Finney had been a respected member of the Cypherpunk community. There were numerous advantages to tapping into these networks: members of both lists were likely technologically sophisticated enough to pseudonymously operate nodes, were likely familiar with the rich history of attempts at implementing untraceable electronic payments, and were likely aware of - if not personally concerned with — an erosion of privacy in electronic commerce. Inspiring covert action among them would come naturally. The pre-existing networks' disadvantages, however, were their small size and insular character: growing the network would be daunting.

Nakamoto and Finney departed too early to see the network through to wider adoption, Nakamoto by choice and Finney by circumstance. With their departure and the emergence of Bitcoin service providers, however, Bitcoin tapped into new, wider, and far more influential networks: Silicon Valley, venture capital, and Chinese investors.

Connections to these networks turned Bitcoin from a private communications technology into "fintech," financial technology. Furthermore, there is some irony in considering the 
wider networks within which Bitcoin may be embedded. Bitcoin was designed to be disembedded, at least from the payment system central banks and financial institutions control. That said, forms of embeddedness have emerged in specific, limited cases during Bitcoin's rapid growth. For example, Bitcoin became critical to the illicit Silk Road marketplace and consequently embedded within the global criminal network. Though Silk Road has long been shut down, Foley, Karlsen, and Putniņš $(2019,1798)$ estimates that Bitcoin's criminal footprint has only increased, with an estimated $46 \%$ of annual bitcoin transactions used for illicit activities.

Bitcoin exhibits socialization dynamics at play. Within Bitcoin circles, the term "HODL," a widely embraced mistyping of the word "hold" has come to denote a popular use of Bitcoin: to purchase and hold it for an indeterminate duration, anticipating bitcoin's value to increase substantially. It is at root a speculative activity that has gained a degree of legitimacy among Bitcoin users (Kaminska 2017). Closely related is the pop culture acronym, "FOMO," applied to Bitcoin. "Fear of missing out" characterizes users who, uncertain of Bitcoin's prospects, emulate peers by buying Bitcoin. Symbolism appears to play a socializing role. While there is no physical form of Bitcoin (aside from proprietary tokens issued by some enterprising individuals), stories of Bitcoin often accompany a likeness of a golden coin with a large bitcoin symbol, $\square$, surrounded by stylized network graphics. Exposure to such imagery equates the viewer with notions of money. Bitcoin's shadowy origins also evoke an adaptive creation mystery. Its creator wrote little more than a brief white paper and scattered technical commentary, and appointed no authoritative successor. All the same, some Bitcoin proponents invoke 
Nakamoto's spirit to justify and advocate their own visions of Bitcoin (Bitcoin Unlimited 2015).

Bitcoin users form identities and differentiate from one another in the network. The language of "HODL" and FOMO fosters an early adopter sense of identity. Early Bitcoin adopters are a subgroup of pragmatic users, those who primarily consider Bitcoin a financial technology. Other pragmatics include Bitcoin entrepreneurs, investors, and some technologists. By contrast, purists include those primarily devoted to Internet privacy or various political ideologies such as anarcho-capitalism and technolibertarianism (Golumbia 2016). Disputes within the Bitcoin network, most prominently a technical dispute regarding Bitcoin's scalability, lead to the most recognizable forms of differentiation. The scaling debate has even led to several blockchain "hard forks," creating all-new blockchains branching from the original and running on modified code (known as Bitcoin XT, Bitcoin Unlimited, Bitcoin Classic, and Bitcoin Cash).

\section{Links}

This section focuses on links within the Bitcoin network as flows of information. As information flows, links are the mechanisms by which user identities may be disclosed, making individual behavior subject to third-party monitoring. Links also provide valuable information to service providers: identities and transaction histories may be monetized for advertising.

Two types of links characterize typical information flows in Bitcoin: peer-to-peer and peer-to-service. Peer-to-peer relationships are brief, only existing when a transaction takes place. Peers need not identify themselves other than by their pseudonymous bitcoin 
addresses that enable their interaction. All the same, once such an interaction takes place and is written to the distributed ledger, it is available for all to see. Peer-to-service relationships, such as when a user joins a mining pool or exchange, are more enduring than peer-to-peer relationships because they involve membership or subscription within an organization. Requirements for identifying oneself to a service differ. In the case of a peer-to-peer mining pool, for example, pseudonymous membership is possible, whereas custodial exchanges increasingly require their users to disclose their personal identities to comply with KYC and AML/CFT regimes. Similarly, depending on the service's form of organization on the network, transaction information may be posted to the distributed ledger or it may be aggregated with other transactions occurring within the service and subsequently posted to the ledger.

Information flows are at the heart of Bitcoin's collective action dilemma. For Bitcoin to subvert state authority, its users must take sufficient precautions to ensure their pseudonymity. Because the blockchain records transaction histories, the disclosure of even a relatively few identities may be enough to identify many more through sophisticated deanonymization techniques (Avdoshin and Lazarenko 2018, 91-95). However, pseudonymity comes at a cost. Bitcoin service providers offer user-friendly one-stop interfaces, whereas operating pseudonymously on the network requires a degree of technical aptitude and patience. Bitcoin's validation protocol also poses information processing drawbacks: a ten-minute block validation parameter pushes urgent transactions off the network and onto service providers' platforms. 


\section{Bitcoin's Network Structure}

Bitcoin's social network structure has dramatically changed over time, with consequences for effective collective action. Service providers' emergence and subsequent dominance exemplifies node centrality, leading to what $\operatorname{Kahler}(2009,112)$ describes as "disguis[ed] effective hierarchy." Brokerage between the Bitcoin network and wider social movements or networks has been limited. There are also signs of network segmentation.

During Bitcoin's formation stage, the network comprised individual, mutually connected user nodes. Considering information flows, the network had a low total degree of centrality because each node was connected with one another. As service providers entered, they became central nodes because their users are not directly connected to other users, but are connected via their links to service providers. Rauchs et al. $(2018,33)$ estimates that one in four users connected via service providers as of September 2018, up from one in ten as of December 2016. There is little reason to suggest the trend has abated. Node centrality in Bitcoin increases the influence of figures operating Bitcoin services. Consider the CEOs of two of Bitcoin's largest exchanges: Changpeng Zhao (Binance) and Brian Armstrong (Coinbase). As proxy measures of influence within the Bitcoin network, Changpeng's Twitter feed has 510,000 followers and Armstrong's has 330,500. Compare those figures with two of Bitcoin's most recognized Cypherpunks, developers Nick Szabo (246,000 followers) and Adam Back (191,700). It turns out the executives have become the world's informal spokespersons for Bitcoin and its users. In tandem, such executives have become the new informal sources of authority within the network. 
Original leaders in the Bitcoin network failed to develop links with wider social movements and networks. They could have created links with like-minded privacy advocates like the Electronic Privacy Information Center, the Center for Democracy and Technology, and the Electronic Frontier Foundation. Nonetheless, some outsiders have taken an instrumental view of Bitcoin and are brokering links between the network and outside groups. For example, economist Steve Hanke has organized a philanthropic campaign to raise bitcoin donations and distribute them to recipients in Venezuela, called Airdrop Venezuela (Garcia-Navarro 2019).

The global Bitcoin network has also become internally and externally segmented. Internally, the programmers who improve and keep Bitcoin running are concentrated in a few countries. A proxy for developer location is the distribution of active, full Bitcoin nodes running the latest protocol. As of May 12, 2020, a majority of the 1,867 such nodes were located in Western Europe or the US. Externally, the global distribution of active Bitcoin nodes is also concentrated, with over $55 \%$ of nodes located in the US, Germany, France, and the Netherlands; and another 8\% connecting from China, Russia, and Singapore (Yeow 2020).

Implications of Bitcoin as an Actor in Global Affairs

Taken together, Bitcoin's social network characteristics illuminate a shift from the collective pursuit of its political purpose to one of economic imperatives. Whereas Bitcoin's political purpose is to subvert state authority via untraceable electronic cash, its speculative appeal — intensified by service providers' profit motive — has diminished its potential as a political actor in global affairs. For Bitcoin to subvert state authority, its 
users must participate pseudonymously. In practice, however, service providers' convenience, speed, and familiarity has pulled increasing numbers of users into their hands. This erodes Bitcoin's pseudonymity, making an increasing share of the network subject to state monitoring and enforcement. Nonetheless, the dual economic imperatives of user growth and profit-seeking enterprise have led from obscurity to a $\$ 163$ billion cyber industry in just over eleven years (CoinMarketCap 2020).

Admittedly, while the Bitcoin network as a whole has failed to achieve its political purpose, a substantial portion of the network chugs along, providing the shield of pseudonymity to many users. The network appears to be succeeding in this respect, at least for the criminals who use it in the aforementioned estimated $46 \%$ of annual bitcoin transactions (Foley, Karlsen, and Putniņš 2019, 1798). However, criminals have greater incentives - and thus are likely to go to greater lengths - to operate covertly than the typical bitcoin user. The costs of pseudonymity are a small price for criminals to pay compared to the risk of being identified. Another possibility is that state actors may use bitcoin to avoid economic sanctions. Cybersecurity firm FireEye speculates that in early 2017, North Korea responded to increased US sanctions by stealing bitcoin from several South Korean exchanges (McNamara 2017). Finally, Bitcoin may offer terrorists a way to covertly fund their operations. This may ultimately be the case, but Cynthia DionSchwarz, David Manheim, and Patrick B. Johnston $(2019,13)$ finds that "most terrorist groups are currently constrained in their ability to use cryptocurrency because of the limited acceptability and usability of these currencies in the regions in which terrorist groups operate." 
It is also worth probing the claim that Bitcoin has a political purpose to begin with. Recall that this analysis roots Bitcoin's political purpose in its attempt to create electronic cash not subject to state authority. While the Bitcoin white paper does not explicitly make the claim, this analysis interprets "trusted third party" - which the white paper thoroughly denounces - to include the state. By introducing an electronic payment system wholly independent of national monies and financial intermediaries, Bitcoin rejects their value and usability. Furthermore, had Bitcoin been intended merely as an innovative electronic payment system, there would have been no need to incorporate pseudonymity. Indeed, there have been countless payment innovations since 2008, from peer-to-peer transaction systems such as Venmo and Zelle to digital wallets such as Google Pay. Each of which, however, obtain users' identities and are fully integrated with the financial system, making them subject to state authority.

This analysis highlights several opportunities Bitcoin missed in promoting collective action. While the emergence of service providers may have been inevitable given Bitcoin's profit-making potential, a few organizing principles may have offset their influence. First, had the agenda been more fully formed at the outset, it may have been less prone to contestation and dilution. Nakamoto may not have been able to foresee the full course of Bitcoin's social development, but left considerable room to reinterpret its original purpose. Second, norm diffusion and identity formation in the Bitcoin network have worked against its political purpose. Had Nakamoto explicitly and comprehensively written political principles into Bitcoin's founding documentation and seen them through the network's early growth, Bitcoin norms and identities may have taken a more political character. Finally, increased brokerage may have counteracted the rise of profit-seeking 
enterprises' informal leadership. For example, linking to privacy-oriented networks outside Bitcoin could have drawn more pseudonymous users into the network, increasing their share relative to those participating through service providers. 


\section{Chapter 3: How Does Bitcoin Work?}

Bitcoin is an electronic cash system that, because it is intended to be untraceable, must operate independent of incumbent financial institutions and monetary authorities. Additionally, because it must be able to operate over the Internet, it cannot be based on a commodity such as gold or silver. How does Bitcoin operate independent of banks, monetary systems, and commodities? This chapter describes how Bitcoin's solution, blockchain technology, works, and conceptualizes the blockchain as a public good that provides services traditionally ascribed to incumbent financial institutions, monetary systems, and commodity money.

\section{Blockchain Technology}

Bitcoin is built on two technological innovations known as "distributed ledger technology" (DLT) and "blockchain.” Bitcoin was not the first digital currency, but DLT and blockchain made it the first to resolve a "double-spending problem" inherent to digital currency without the need of a third-party intermediary. The double-spending problem surfaced because unscrupulous users could replicate digital currency for reuse and abuse. Nakamoto's decentralized transaction verification system also provided for dispute settlement without the need of a third party. By removing the need for third party mediation, cryptocurrency provides for transaction security while eliminating the transaction costs associated with such mediation. The implications are widespread, as 
counterparties to transactions can be assured trust in one another without the need for third parties like banks, marketplaces, brokers, insurers, or even adjudicators. ${ }^{9}$

DLT leverages computer networking to create and maintain a decentralized transaction ledger. Prior to DLT, digital ledgers were accessed through computer networks but the data itself was centralized in a single database. Consider logging in to your bank's online banking service and viewing the transactions stored in their database. With DLT, every node (user) in the network maintains a separate instance of the ledger that is synchronized at all times with every other node's instance (see Figure 3). William Mougayar (2016) presents shared Google Docs or Sheets, in which the information contained is synchronized among multiple users simultaneously, as a helpful analogy of DLT. No single party controls the ledger, making a DLT ledger robust because it is not subject to failure at any single point, in contrast to conventional databases.

Nonetheless, a subset of parties manages the platform upon which the ledger operates. Herein lay the key power dynamic of DLT, as the parties managing the platform (typically its associated developers and influential nodes) set the rules of behavior for the related technology underpinning cryptocurrency: the blockchain.

${ }^{9}$ The implications of eliminating such transaction costs through DLT go well beyond this paper's focus on cryptocurrency, including applications for voting systems, property registries, prediction markets, and pharmaceutical authentication to name but a few. 
Figure 3. Centralized and Distributed Ledgers
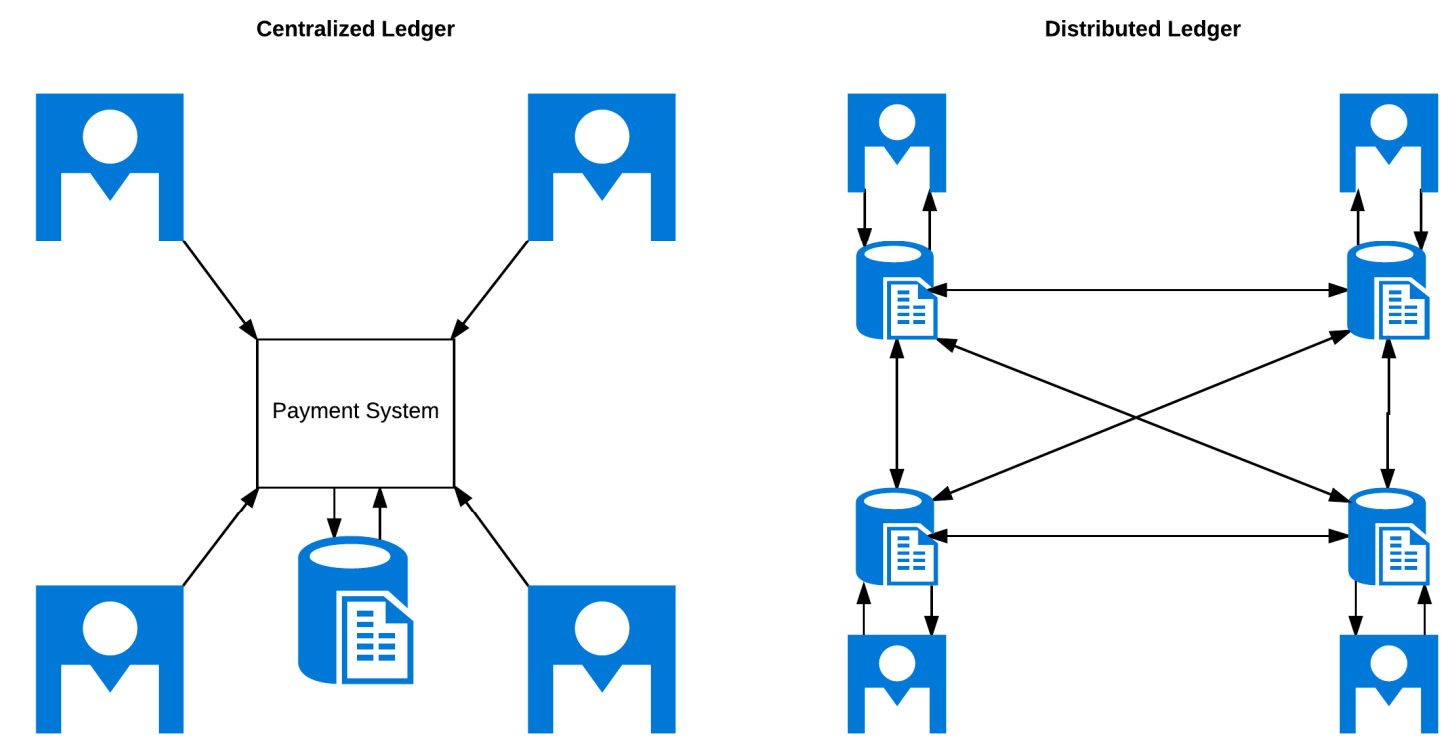

Source: Santander InnoVentures $(2015,14)$.

The idea behind blockchain was developed well before Nakamoto employed it in Bitcoin, by Stuart Haber and W. Scott Stornetta, researchers at Bell Communications Research. Observing that information was increasingly becoming digital and subject to modification, Haber and Stornetta (1991) proposed a method of computationally timestamping digital media that would result in an immutable record; or at least, a record in which modification could be easily detected. We now call this blockchain. Wessel Reijers, Fiachra O’Brolcháin, and Paul Haynes $(2016,136)$ defines blockchain as “a public record of time-stamped transactions that is reinforced by the computational efforts of the decentralized network of 'miners' (people controlling computational nodes that are validating transactions)." 
A blockchain comprises linked collections of "blocks" which in turn are collections of transactions validated in a network within a given timeframe. ${ }^{10}$ No single block is ever removed from the chain, meaning that all blocks - and the transactions validated within — are available for public scrutiny back to the blockchain's genesis. This provides a publicly available, immediately accessible audit trail. A given blockchain may constitute monetary transactions, as in the case of cryptocurrency, or nonmonetary transactions such as contracts or even collective decisions. While anyone may audit a blockchain's transaction history, not all transaction information is publicly revealed (such as the identities of parties to a transaction). Cryptocurrency software analogous to a wallet allows users to reveal their cryptocurrency balance to the network without identifying themselves. By use of these "wallets," monetary transactions occurring along the blockchain involve validating the availability of cryptocurrency in a sender's wallet, subtracting the agreed amount from that wallet, and adding that amount to the receiver's wallet.

One of the challenges to running a cryptocurrency on a distributed network is to determine how the money supply adjusts over time. Since there is no monetary authority or banking system involved in Bitcoin, Nakamoto had to devise a set of rules to govern the supply of bitcoin. David Yermack $(2015,34)$ writes that Bitcoin's protocol "provides

\footnotetext{
${ }^{10}$ Bitcoin uses a ten-minute timeframe to group validated transactions into one block and timestamp it accordingly. A block comprises a set of validated transactions that are each encrypted into a unique "hash value." Any change to a transaction will change its hash value and invalidate the transaction, providing for integrity without compromising user identity. The collection of hash values becomes a block's "header," which is timestamped and includes a hash of the previous block's header along with an arbitrary number called a "nonce." This header becomes part of a complex, cryptographically generated mathematical puzzle for which the nonce is used to find the solution through trial and error. Puzzle-solving nodes in the network ("miners") set to work attempting to find a solution. Once a solution is found, the other nodes in the network double check it and, if confirmed, add the block to the previous block, forming the blockchain. All nodes' ledgers are consequently updated.
} 
for the rate of seigniorage to slow asymptotically to zero by the year 2140, when the last bitcoin is scheduled to be released and the final total will be fixed at 21 million units." In practice, new bitcoins are created each time a miner validates a block by solving the aforementioned, randomly generated cryptographic puzzle. The rate at which bitcoins are created is coded to halve every four years, so the current "block reward" equals 6.25 bitcoins. The next halving is expected in 2024. The block reward is a critical incentive for nodes to contribute computing power to the network, used for authenticating transactions and maintaining the ledger. While the fixed total supply and scheduled rate of growth are coded into the Bitcoin protocol, they could be changed if a majority of the network's computing power adopted the change.

Bitcoin's implementation of DLT and blockchain technology has gained notoriety for applications that go beyond electronic cash. Its decentralized transaction verification system provides for dispute settlement without relying on a third party. By removing the need for third party mediation, Bitcoin provides for transaction security while eliminating the transaction costs associated with such mediation. The implications are widespread, as counterparties to transactions can be assured trust in one another without the need for third parties like banks, marketplaces, brokers, insurers, or even adjudicators. Theoretically, any transactional relationship occurring online can be validated or enforced within the network without the need for a third party. Practical applications include voting systems, property registries, prediction markets, and pharmaceutical authentication to name but a few. According to MIT's Digital Currency Initiative (2020):

Many observers have compared the potential of blockchain technology with that of the Internet. The Internet enabled people to easily call each other without a phone company, send a document without a mail carrier, 
or publish an article without a newspaper. As a result, today more than 2.9 billion people depend on that decentralized communications protocol to more efficiently communicate with one another.

Similarly, the blockchain - the underlying technology behind Bitcoin and other cryptocurrencies - is a decentralized public ledger of debits and credits built on the principle that no one person or company owns or controls it. The intent is that users control it directly. This new system promises to let people transfer money without a bank, write simple, enforceable contracts without a lawyer, or turn personal property such as real estate, digital music, or a household's rights to locally generated solar energy into digital assets that can be transferred or pledged with near-zero transaction fees. Many are projecting that the impact from what is being called the "Internet of Value" will be similar to that of the "Internet of Information"- - disrupting traditional industries, challenging existing regulations, and significantly increasing the volume of commerce by both dramatically lowering the cost to transact and allowing two parties to enter into exchanges without trusting each other.

\section{Blockchain as a Public Good}

Blockchain is Bitcoin's novel solution to the double-spending problem inherent in disintermediated electronic payment systems. Prior to Bitcoin, electronic payment systems (such as ACH, credit cards, and even David Chaum's DigiCash) necessitated third-party intermediation. Financial institutions such as banks were necessary to maintain transaction ledgers, settle account balances, and secure deposits. Monetary authorities were necessary to manage the currency and money supply. Furthermore, a legal framework and set of institutions, including courts and law enforcement, were necessary to uphold property rights and adjudicate disputes. Blockchain makes Bitcoin's electronic cash system possible without necessitating such financial institutions, monetary authorities, and legal institutions.

One way to think of blockchain, then, is as a regime of rules and assurances that provide for accounting, minting, and security. In other words, the blockchain assumes the functions of conventional third-party intermediaries through technical processes. DLT 
maintains a permanent, immutable, and auditable public account of all transactions conducted in the system. Transactions are written to the distributed ledger through the blockchain process, at the core of which is mining. Mining operates according to cryptographically-based rules coded into the system's protocol, as well as a set of assurances between and among miners and users. Miners are compelled to only post valid transactions to their block, for example, because other miners will ultimately reject their block if it includes invalid transactions. Newly-minted cryptocurrency is added to the system as a function of mining: in Bitcoin's case, a miner currently receives 6.25 newly created bitcoins for successfully mining a new block, increasing the total money supply within Bitcoin. Finally, the key to Bitcoin security is ensuring that no single miner persistently controls more than $50 \%$ of network processing power. Controlling over $50 \%$ of network processing power over time would allow a miner to write double-spent bitcoin transactions to the blockchain, also known as a "51\% attack." To prevent this, Bitcoin's consensus protocol encourages miners to compete with one another, reducing the chance that any single miner would persistently attain a majority of processing power (see the Technical Description of Bitcoin in Chapter 2).

In the following analysis, I conceptualize blockchain — a regime of rules and assurances that provide for accounting, minting, and security —as a public good. It meets the definition of public goods because no-one with Internet access can be effectively excluded from using the blockchain, and any user's consumption of blockchain (i.e., use of blockchain services) does not reduce its availability to other users. By contrast, bitcoin, the unit of cryptocurrency, is a private good because each unit of bitcoin is exclusively owned and used by private parties at will. To clarify the conceptualization, it is worth 
distinguishing blockchain and bitcoin from two other common types of goods: club/toll goods and common pool resources. Club goods (also known as toll goods) are goods by which parties may be effectively excluded from use, but any single party's consumption of the good does not reduce its availability to others. Toll roads, movie theaters, and child care centers are examples of club goods. Common pool resources are goods that are difficult to exclude parties from using, but consumption by one party reduces its availability to others, such as public fishing stocks and forests.

\section{Bitcoin's Public Goods Problem}

Bitcoin's blockchain represents a novel solution to the classic public goods problem. The public goods problem arises when a public good is underprovided because individual members of a collective do not have sufficient incentive to contribute to its provision. Since individuals can benefit from the good whether or not they contribute, they have a strong incentive to "free-ride," or refrain from contributing. In the case of Bitcoin, the blockchain requires substantial computing resources to function. These resources must be provided voluntarily by enough users to prevent a $51 \%$ attack (specifically, enough that no single party contributes a majority of resources). Users may benefit from the blockchain whether or not they contribute computing resources; noncontributing users are free-riders. Consequently, Bitcoin's public goods problem is securing the necessary computing resources to provide the blockchain, while many users are likely to free-ride. If too many free-ride, the resources are not provided and the entire blockchain fails. 
Bitcoin's solution to the free-rider problem involves providing selective incentives and overcoming a collective mining threshold. Bitcoin offers a "mining reward," denominated in bitcoin, to users who choose to contribute (now "miners"). The reward to any individual miner is probabilistic, generally decreasing in probability as more miners compete for the reward. ${ }^{11}$ It is, in the tradition of Mancur Olson, a "selective incentive" and a private good. However, because the reward is denominated in bitcoin, its value is tied to the success or failure of the network as an electronic cash system. In turn, the network's success as an electronic cash system is a function of whether or not it provides the public good (a functioning blockchain). So if the public good is provided (i.e., enough individuals are miners), the mining reward has some value; however, if the public good is not provided, the mining reward has no value. Selective incentives alone are not sufficient to overcome the free-rider problem; the number of individuals who mine must exceed a particular threshold for the entire system to become viable. The concept of thresholds in collective behavior incorporates a second research tradition to the analysis, stemming from Thomas Schelling's work on "tipping phenomena" (or “tipping points").

\section{Selective Incentives, Tipping Points, and Thresholds}

Successful collective action is a formidable challenge to concerted human endeavor. A wide and diverse literature, particularly in political science and economics, considers "collective action problems." For Thomas Hobbes ([1651] 1965, 94-98) life in

\footnotetext{
${ }^{11}$ However, individual miners may compensate for their declining probability of winning by deploying more powerful mining "rigs" or joining cooperative mining pools.
} 
the "natural condition of mankind" is "nasty, brutish and short," a condition of "war of every man against every man.” This state of nature is only overcome when individuals submit to a common authority that preserves their security. Humans will not naturally cooperate to coexist peaceably, then, but must be imposed upon to do so by a third party. Alternatively, Jean Jacques Rousseau $([1761], 105)$ employs a hypothetical "stag hunt" in which a group of individuals would each be better off cooperating to capture a deer, but each faces the temptation to defect for a surer, but less wholesome meal:

Was a deer to be taken? Every one saw that to succeed he must faithfully stand to his post; but suppose a hare to have slip[ped] by within reach of any one of them, it is not to be doubted but he pursued it without scruple, and when he had seized his prey never reproached himself with having made his companions miss theirs.

To Rousseau, the collective action problem is a matter of trust and coordination: if each hunter can trust every other hunter to coordinate their actions, they can all be better off by capturing the deer together.

In Mancur Olson's (1965) work, public goods present an especially intractable type of collective action problem. Paul Samuelson $(1954,387)$ first distinguishes public goods, or "collective consumption goods," from private goods. A public good is both non-excludable and non-rivalrous. Non-excludability means that anyone can consume the good, and non-rivalry means that one individual's consumption of the good does not reduce its availability to others. Addressing public goods, Mancur Olson (1965, 2) reframes the collective action problem, whereas "unless the number of individuals in a group is quite small, or unless there is coercion or some other special device to make individuals act in their common interest, rational, self-interested individuals will not act to achieve their common or group interests." When a group attempts to provide goods 
that are both non-excludable and non-rivalrous, individuals in the group have strong incentives to "free ride." That is, especially as the group grows larger in number, any individual can choose not to contribute to provision of the public good as long as there are many who contribute. The problem arises when too many individuals free-ride, resulting in under-provisioning of the public good. Olson's (16) solution is that "large organizations that are not able to make membership compulsory must also provide some noncollective goods in order to give potential members an incentive to join.” These “noncollective goods" are also known as "selective incentives."

While Olson was developing his theory of collective action-based largely on assumptions of rational, self-interested individuals and material incentives-Thomas Schelling began probing collective action from a different approach: that of individuals' beliefs and the interdependence of their interactions with others. Examining segregation in US neighborhoods, Schelling (1971) discovers a "tipping phenomenon" whereby individuals' preferences to live next to individuals of the same race, even admitting some level of racial diversity, can ultimately lead to total segregation. That is, beyond a certain level of diversity, the "tipping point," individuals will self-segregate by moving out of diverse areas into segregated neighborhoods. Schelling $(1978,102)$ generalizes his original findings of tipping to other collective behaviors, analogizing that:

suppose that for some activity [...] there are some people who will attend regularly no matter what the attendance is and some who will never attend, but most people will attend if, but only if, enough others do. Everybody has his own definition of how many is "enough." And it can mean either enough to make it interesting or enough to make it imprudent to be absent.

Whereas a tipping point "is nearly universal and irreversible, or alternatively [...] accelerates a process that is inevitable and irreversible anyway" (Schelling 1971, 182), 
the related concept of a "threshold" is used to describe a such a phenomenon that may be reversed if the number of actors falls below a minimum proportion. For example, ChienChung Yin (1998) applies the concept of thresholds to explain differing patterns of political protest.

\section{The Would-Be Bitcoin Miner Game}

In this section, I present a game-theoretical model of Bitcoin mining that incorporates selective incentives and a threshold. The model differs from most gametheoretical models of Bitcoin mining in that it focuses on the initial decision to mine (or not), rather than on mining behavior once that decision has already been made. In doing so, the model provides insight into how Bitcoin manages to extract resources for the provisioning of public goods. The core contribution of this model is describing how networked forms of organization can provide public goods by offering selective benefits once a threshold is surmounted.

Game-theoretical modelling has become commonplace in analyses of Bitcoin and blockchain technology. Nicolas Houy $(2016,54-55)$ examines miners' incentives to include transactions in any particular block as a function of time vis-a-vis other miners and probabilistic rewards with transaction fees. Houy's game-theoretical model, a "speed game," posits that "the smallest block possible for both miners is the only Nash equilibrium of the Bitcoin mining game" (58). Nicola Dimitri (2017, 31-32) characterizes Bitcoin mining as an "all-pay contest," finding that "the intrinsic structure of the mining activity seems to prevent the formation of a monopoly, because in an equilibrium with two miners, both of them will have positive expected profits for any 
level of the opponent's costs." Yoad Lewenberg et al. $(2015,3)$ models Bitcoin miningin particular, users' choices to join and switch mining pools — as a "transferable utility coalitional game," wherein "the value of a coalition depends solely on the members of that coalition, with no dependence on the other players." Game theory also provides insight into when users would decide to attack the network or other users. Ziyao Liu et al. (2019) surveys the literature on game-theoretical analysis with respect to blockchain security, finding 25 different models of attack games and other security issues, of which 12 take the non-cooperative game form and 18 are related to compromising the mining process. Benjamin Johnson et al. (2014) model Bitcoin mining pool behavior as a competitive game in which pool operators choose between investing their computational resources in mining or attacking other pools through DDoS. ${ }^{12}$ They find that pools with lower hashrates ${ }^{13}$ have stronger incentives to attack pools with higher hashrates than to devote their resources to mining.

While game theory has been used to describe different types of strategic behavior with respect to Bitcoin and blockchain, game theory has not yet been applied to the question of public goods provision. Indeed, reflecting on the puzzle as to why Bitcoin miners are not in practice playing Nash equilibrium strategies, Houy $(2016,62)$ suggests that further research into "the Bitcoin mining game should include a supplementary public good game on top of it." This section applies game theory to model Bitcoin's provision of blockchain as a public good. The mining process is essential to providing the blockchain to all users, but as Houy demonstrates, the basic Bitcoin mining game's Nash

\footnotetext{
${ }^{12} \mathrm{DDoS}$ is short for "distributed denial of service," an attack intended to disrupt a computer or network resource by overwhelming it with data sent from many different computers at once.

${ }^{13}$ Hashrate is a measure of computing power in the Bitcoin network.
} 
equilibrium solution leads to under-provisioning of the resources upon which blockchain depends. In Houy's basic Bitcoin mining game, miners' best strategy is to minimize the number of transactions they include in a block. Houy shows that by doing so, miners can increase the probability that their mined block propagates through the network first, earning the mining reward. The result is that Bitcoin transaction processing would slow to a trickle. This has not been the case, in practice. How, then, does the network persist? The following model extends Houy's analysis by incorporating the belief users have of whether or not the mining threshold is met into their expected payoffs when deciding to mine or not. Doing so not only brings the game-theoretical model of mining into correspondence with real-world behavior, but it also tells us something about how the network form of organization can facilitate solutions to coordination problems.

The model presented here takes a step back from Houy's analysis — indeed from the analyses of many game-theoretical works on Bitcoin mining to date - and addresses the issue of why a user would mine bitcoin in the first place. On the one hand, encouraging mining is at the heart of Bitcoin's coordination problem. To provide the public good of the blockchain, Bitcoin must procure computing resources from a distributed set of users to whom there is no authority to compel. Alternatives, such as provisioning through private enterprise or nonprofit organization would introduce thirdparty intermediaries to the system. The result would be entirely at odds with Bitcoin's purpose. On the other hand, Bitcoin's vulnerability to a 51\% attack compels the competitive logic of Bitcoin mining: Bitcoin must incentivize enough users to mine so as to prevent any one miner from securing a majority of hashrate over time. Put differently, the free-rider problem is complicated in Bitcoin by the addition of a threshold. 


\section{Modelling Bitcoin Miner Behavior}

Executing the Bitcoin protocol relies on the incentive structure of individual miners. Miners are the nodes that validate and secure Bitcoin transactions while engaging in the process of monetary expansion. They are critical to Bitcoin network services and security. The possibility of receiving newly-minted bitcoin as well as transaction fees associated with transactions incentivizes miners to contribute computing power to the network. Such rewards are payoffs in a game-theoretical model, for which miners (players) compete with one another to attain. Each miner (player) has a set of options to engage in the competition, known as strategies. The following game-theoretical Bitcoin mining models are based on three key assumptions: 1) a miner's payoffs incorporate the totality of that miner's concerns for each possible outcome of the game, 2) a miner has full knowledge of the structure of the game, and 3) a miner acts rationally to choose the strategy that will maximize their payoff given that miner's beliefs about the choices of other miners.

The following two would-be Bitcoin miner games model how Bitcoin solves its collective action problem by incentivizing users to become miners, subject to beliefs about whether or not there are enough other miners to surmount the necessary threshold. It is critical to note how the relative value of a mining reward to the cost of computing power (denominated in conventional currency) complicates the threshold. If the entire network comprises miners (and thus, no "free-riding" users), they might as well simply exchange their hard-earned money with one another rather than go through the trouble of mining. That is, assuming each miner has an equal probability of winning the next mining reward, each individual's portion of newly minted bitcoin is roughly equivalent to every 
other's portion, over time. Why would they waste their computing resources? Once you add "free-riding" users, however, the demand for bitcoin increases compared to its supply, consequently increasing its value to any individual miner. This situation begins to make mining rewards lucrative compared to the costs of mining. Interestingly, once the mining threshold is overcome (and thus blockchain functions for all users, regardless of whether or not they mine) free-riding appears to make selective benefits more valuable and mining becomes worthwhile. To model this dynamic, I present two games that models users' choices to mine bitcoin or not, incorporating users' beliefs regarding whether or not the mining threshold will be met.

The would-be mining game is a simultaneous, global game, assuming that more than two would-be miners exist at any given time. That is, the total number of nodes (users) in the Bitcoin network is greater than two, of whom any may choose to mine or not. The global Bitcoin mining game has $n$ players, $\{1,2, \ldots, N\}$, each with a set of possible strategies. The game's outcome (or joint strategy) is the set of strategies chosen by all players. Each player $i$ has a payoff function $P_{i}$ mapping the game's outcomes to a numerical payoff for $i$. For each outcome of strategies $\left(S_{1}, S_{2}, \ldots, S_{n}\right)$, Player $i$ has a payoff $P_{i}\left(S_{1}, S_{2}, \ldots, S_{n}\right)$. Consequently, strategy $S_{i}$ is a best response of Player $i$ to the set of strategies chosen by all other players, $\left(S_{1}, S_{2}, \ldots, S_{i-1}, S_{i+1}, \ldots, S_{n}\right)$, if:

$$
P_{i}\left(S_{1}, S_{2}, \ldots, S_{i-1}, S_{i}, S_{i+1}, \ldots, S_{n}\right) \geq P_{i}\left(S_{1}, S_{2}, \ldots, S_{i-1}, S_{i}^{\prime}, S_{i+1}, \ldots, S_{n}\right)
$$

for all other possible strategies $S_{i}^{\prime}$ available to Player $i$. An outcome consisting of the set of strategies $\left(S_{1}, S_{2}, \ldots, S_{n}\right)$ is a Nash equilibrium if each strategy is a best response to all others. 
Assuming all players' hashrates (computing power) are equivalent to one another, and given $n$ players, the probability that Player $i$ mines the next block is expressed by $\sigma(i)$ where the probability of all $N$ possible outcomes is represented by the probability vector:

$$
\sigma=(\sigma(1), \sigma(2), \ldots, \sigma(N)) \text {, with } \sigma(i) \geq 0 \text { and } \sum_{i}^{N} \sigma(i)=1 \text {. }
$$

Each model assumes that the value of mining rewards in bitcoin, $b$, exceeds the cost of mining, $c$, and that mining rewards and cost do not vary by player. So when faced with the choice to mine or not, Player $i$ considers their belief regarding whether or not the mining threshold will be met, $\gamma$, where $\gamma=1$ if the threshold is met and $\gamma=0$ if not met, the probability of winning the next mining reward, $\sigma(i)$, multiplied by its value in bitcoin, $b$, minus their cost to mine, $c$ :

$$
P_{i}=\gamma \cdot \sigma(i)(b)-c
$$

To begin, consider a game in which Player $i$ does not believe the threshold will be met. Each player has two possible strategies to choose from: 1) mine, which is to say, cooperate in the provisioning of the public good of blockchain, or 2) not mine, which is to say, defect from provisioning the public good. The public good is provided only if both players choose to mine, from which all players - whether or not they choose to minemay benefit. In this scenario, Player $i$ faces the simple payoff, $P_{i}=\gamma \cdot \sigma(i)(b)-c$. This "Would-Be Bitcoin Miner Game, Threshold Believed Not Met" (Model 1) exhibits the following payoff matrix, with payoffs represented in order of preference: 
Figure 4. Would-Be Bitcoin Miner Game Payoff Matrix, Threshold Believed Not Met Any Other Player, $\{2, \ldots, N\}$

\begin{tabular}{|c|c|c|c|}
\hline & & $\begin{array}{c}\text { Mine } \\
\text { (Cooperate) }\end{array}$ & $\begin{array}{c}\text { Do Not Mine } \\
\text { (Defect) }\end{array}$ \\
\hline \multirow{2}{*}{ Player 1} & $\begin{array}{c}\text { Mine } \\
\text { (Cooperate) }\end{array}$ & 1,1 & 1,2 \\
\hline & $\begin{array}{l}\text { Do Not Mine } \\
\text { (Defect) }\end{array}$ & 2,1 & 2,2 \\
\hline
\end{tabular}

At a payoff of (2), the best outcome for both players results from the choice to not mine, regardless of what the other player does, avoiding the lost cost of computing power in the event the threshold is not met (where $\left.\gamma=0, P_{i}=0 \cdot \sigma(i)(b)-0\right)$. The payoff (1) to either player of mining $\left(\gamma=0, P_{i}=0 \cdot \sigma(i)(b)-c\right)$ represents a loss of $c$. Consequently, each player has a strictly dominant strategy of not mining, meaning that choosing to not mine is better than choosing to mine, regardless of what the other player chooses. The pair of strategies is known as a Nash equilibrium because regardless of what the other player does, neither player has an incentive to deviate from their strategy. It is also the best response to any strategy the other player chooses.

Next, consider the scenario wherein Player $i$ does believe the threshold will be met.. Again, each player may either mine (cooperate) or not mine (defect). Player $i$ now faces the payoff where $\gamma=1$, or $P_{i}=1 \cdot \sigma(i)(b)-c$. The following payoff matrix comprises the relative payoffs and possible strategies of each player in the "Would-Be Bitcoin Miner Game, Threshold Believed Met” (Model 2): 
Figure 5. Would-Be Bitcoin Miner Game Payoff Matrix, Threshold Believed Met Any Other Player, $\{2, \ldots, N\}$

\begin{tabular}{|c|c|c|c|}
\hline & & $\begin{array}{c}\text { Mine } \\
\text { (Cooperate) }\end{array}$ & $\begin{array}{l}\text { Do Not Mine } \\
\text { (Defect) }\end{array}$ \\
\hline \multirow{2}{*}{ Player 1} & $\begin{array}{c}\text { Mine } \\
\text { (Cooperate) }\end{array}$ & 2,2 & 2,1 \\
\hline & $\begin{array}{l}\text { Do Not Mine } \\
\text { (Defect) }\end{array}$ & 1,2 & 1,1 \\
\hline
\end{tabular}

With a relative payoff of (2), each player's best choice is now to mine, regardless of what the other player chooses. In this case, the player maximizes their probability of winning the mining $\left(P_{i}=1 \cdot \sigma(i)(b)-c\right)$. A relative payoff of (1) results from the choice not to mine, regardless of whether or not the other player chooses to mine. Here, the player forgoes both the possible mining reward and the cost of mining $\left(P_{i}=0\right)$. Each player now has a strictly dominant strategy of mining, resulting in a Nash equilibrium solution. Finally, because the game's cumulative relative payoff is the greatest at its Nash equilibrium, it is also a socially optimal solution.

\section{Bitcoin Mining, Observed}

How well do the would-be miner game models mirror actual Bitcoin mining behavior? Model 1 predicts that users would not mine bitcoin as long as they believe the mining threshold will not be met. By contrast, Model 2 predicts that users would mine as long as they believe the mining threshold will be met. In observing that Bitcoin has been operative since 2009, with its blockchain functioning and having not been subjected to a $51 \%$ attack, Model 2 is more reflective of actual behavior. Nonetheless, it is worth 
considering how many miners Bitcoin attracts in the first place. While miners are not obligated to identify themselves, some do, enabling a minimum count of miners participating over time. Mining a block creates a special transaction within the block called the "coinbase transaction." This transaction distributes the block reward and transaction fees to the miner. It is also where the block's miner may append a short message, if they so choose. ${ }^{14}$ Examining the coinbase data, I find that between at least 1 and at least 45 unique miners successfully mined Bitcoin blocks annually, generally increasing over time (see Table 1). Since the results only include self-identified miners ${ }^{15}$ who successfully mined blocks, it is reasonable to assume that the actual number of miners is substantially higher.

Table 1. Minimum Annual Number of Unique Bitcoin Miners, 2009-2019

\begin{tabular}{|c|c|c|c|c|c|c|c|c|c|c|c|}
\hline Year & 2009 & 2010 & 2011 & 2012 & 2013 & 2014 & 2015 & 2016 & 2017 & 2018 & $2019 *$ \\
\hline$\#$ & 1 & 1 & 8 & 15 & 23 & 33 & 43 & 40 & 45 & 40 & 28 \\
\hline
\end{tabular}

* First two quarters, 1/1/2019-6/30/2019

\section{Discussion}

As the game theoretical models and empirical results show, Bitcoin provides blockchain as a public good by coordinating voluntarily provided computing resources by offering selective incentives, the value of which are a function of whether or not users

\footnotetext{
${ }^{14}$ As mining pools - conglomerations of individual miners who combine their computing power to outcompete other miners for rewards - became commonplace in late 2010, many began to voluntarily identify themselves by including their pool name in the coinbase transaction. Since such tags are selfidentified, relying on them to identify pools is subject to error. Nonetheless, pools have a strong incentive to accurately identify themselves as winners of their blocks. Doing so acts as a cost-free form of branding intended to attract new members to their pools and to retain existing members. Canhui Wang, Xiaowen Chu, and Qin Yang (2019) also estimates that the actual rate of intentional mis-representation by pools is insignificant.

${ }^{15}$ Plus all unidentified miners, which were collectively counted as one during each year.
} 
believe the mining threshold will be met. Not only has it succeeded in this respect, but it has also managed to coordinate an unrivaled amount of processing power without a single point of authority. To put the precise level of processing power in perspective, Melanie Swan $(2016,189)$ estimates, "All of the worldwide computers running the Bitcoin mining operation collectively comprise the world's biggest and fastest supercomputer. [...] Comparisons could be made with Google, who is estimated to have 10 million servers comprising one PetaHash.” Bitcoin surpassed the $1 \mathrm{PH} / \mathrm{s}$ benchmark in 2013 and operated at approximately 71,000 PH/s on August 14, 2019. As prior research has shown, Bitcoin accomplishes this feat in part through incentives crafted-and embedded in its technical design - to capitalize on the rational self-interest of prospective miners. Moreover, Bitcoin's blockchain—specifically, whether or not it secures the resources needed to function - plays a fundamental role in how prospective miners evaluate their payoffs to contributing resources. In other words, Bitcoin mining behavior cannot be understood without considering blockchain as a public good.

That said, it is worth considering several alternative explanations of the findings. One possibility is altruism. Miners may contribute resources to Bitcoin because they believe it provides a service to people that would not otherwise be available. Similarly, miners may have normative reasons for contributing: they may simply believe in the cause of electronic cash. In fact, this appears to have been the case for Bitcoin's first year or two of operation. It is indeed possible that some miners are motivated by altruism or particular causes, but it is currently difficult to square with the persistent dominance of profit-seeking mining pools, which have accounted for the vast majority of mining since their emergence. Another possibility is that a hegemonic actor may contribute resources 
in an effort to gain influence over the network. Given the competitive composition of mining, this does not seem to be the case. However, if mining becomes concentrated, the risk to this scenario increases. Perhaps the most plausible alternative explanation is akin to a criminal conspiracy, but is not entirely at odds with the models based on network externalities. In the criminal conspiracy scenario, the Bitcoin network is inherently valuable as a way to conduct illicit transactions. To these illicit actors, therefore, the costs of devoting resources to mining may be worth it as long as the network functions as expected.

Bitcoin presents a situation where the threshold to providing the public good is only met if the ratio of free-riders-to-contributors is sufficiently large, and the number of miners is sufficient enough that no single party contributes a majority of resources. Perhaps surprisingly, given the model presented, as long as the threshold is met to provide blockchain, the number of free-riders may climb toward infinity, without a corresponding increase in the number of miners. In fact, the payoff to mining may tend to decrease as the number of miners grows (owing to marginal effects of increasing individual processing power while the number of competitors increases).

Broadly speaking, the transformative potential of DLT and blockchain technology is that any transactional relationship online can be validated or enforced within such a network without the need for a third party. As an ambitious example, the Ethereum (2019) platform — now the second-largest cryptocurrency behind Bitcoin — claims to go well beyond cryptocurrency and "run smart contracts: applications that run exactly as programmed without any possibility of downtime, censorship, fraud or third-party interference." Some Ethereum developers go so far as to call it "a new kind of law" in 
which "an agreement involving transfer of value can be precisely defined and automatically enforced with the same script" (Etherscripter 2020). 
Chapter 4: Why Does Bitcoin Matter?

This chapter compares Bitcoin with the Cypherpunks, Bitcoin's predecessor in direct cyber action, and considers several implications of Bitcoin and blockchain that are "shaking the system." I argue that Bitcoin has adapted to its twenty-first century context, making it a resilient social movement. Namely, it employs pseudonymity and a distributed network organizational form to withstand attack, while using an effective repertoire and public displays to increase its salience to the public. While many of the network's implications are conditional on its continued growth, given Bitcoin's social movement features and the possibilities of blockchain technology, it is not too early to sketch out some of their most significant potential consequences. Specifically, I consider international capital markets; foreign exchange reserves and currency competition; central bank monetary policy autonomy; crime, terrorism, and sanctions; and systems of trust.

Bitcoin as a Social Movement: A Comparative Analysis

\section{Contentious Politics}

To begin, what constitutes the political? Surely, governments are involved in politics. By this admission, the governed, as counterparts of governments are also involved. But is every act of either party "political"? Supposing a concrete answer to the question of what constitutes the political, the challenge becomes how we observe politics. For the purposes of this analysis, I turn to Sidney G. Tarrow's (2013) concept of “contentious politics.” Tarrow (266) defines contentious politics as: 
episodic, public, collective interaction among makers of claims and their objects when: (a) at least one government is a claimant, an object of claims, or a party to the claims, and (b) the claims would, if realized, affect the interests of at least one of the claimants or objects of claims.

Types of contentious politics, or "collective political struggle," include social movements, riots, protest, strikes, and even "civil wars, revolutions, and episodes of democratization." Notably, contentious politics is at once separate from, but intersects with, "routine political processes-like elections and interest group politics."

Tarrow $(2011,21-22)$ synthesizes a history of study into contentious politics, first identifying four schools of thought: "collective behavior theorists," "resource mobilization theorists," "framing and collective identity theorists," and "political process theorists." To reconcile the findings of the different schools of thought, Tarrow (29-30) argues that "people engage in contentious politics when patterns of political opportunities and constraints change, and then by strategically employing a repertoire of collective action, creating new opportunities, which are used by others in widening cycles of contention." In other words, the emergence of contentious politics depends in part on shifting political circumstances that may be both exogenous and endogenous to the collective actor. Furthermore, Tarrow (22) concludes that successful contention, conceptualized as "sustained interactions with opponents," is a product of "struggles [that] revolve around broad cleavages in society; [that] bring people together around inherited cultural symbols; and [that] build on—or construct—dense social networks and connective structures." 


\section{Social Movements}

Charles Tilly and Lesley J. Wood (2012) traces social movement history since 1768 to define social movements as a particular type of contentious politics. Tilly and Wood (3-4) draws on the work of Doug McAdam, Sidney Tarrow, and Charles Tilly (2001):

treat[ing] social movements as a distinctive form of contentious politicscontentious in the sense that social movements involve collective making of claims that, if realized would conflict with someone else's interest, politics in the sense that governments of one sort or another figure somehow in the claim making, whether as claimants, objects of claims, allies of the objects, or monitors of the contention.

Tilly and Wood (4) argues that social movements are "a major vehicle for ordinary people's participation in public politics," particularly in democratic and semi-democratic regimes.

To distinguish social movements from other types of contentious politics, such as protest, and to elaborate how social movements may lead to collective action without presupposing them as unitary actors, Tilly and Wood (8) derives three core elements of social movements:

(1) campaigns of collective claims on target authorities; (2) an array of claimmaking performances including special-purpose associations, public meetings, media statements, and demonstrations; and (3) public representations of the cause's worthiness, unity, numbers, and commitment [WUNC].

Social movement campaigns, "sustained, organized public effort[s]" distinguish them from sporadic, onetime contentious political events such as petitions or meetings, though they may feature such tactics in a sustained, organized effort. Campaigns "always link at least three parties: a group of self-designated claimants, some object(s) of claims, and a public of some kind." Target authorities may be government officials, but they can 
also include property owners, nongovernmental leaders, and "others whose actions (or failures to act) significantly affect the welfare of many people" (4).

Tilly and Wood (4-5) uses the term repertoire to refer to the second core element of social movements, "an array of claim-making performances." A social movement repertoire includes the tactics it employs, such as the choice of a particular form of organization, venues and types of engagement, and its formation of crosscutting coalition with other organizations. While other political phenomena, such as electoral politics and organized labor, also employ such repertoires, social movements repertoires are distinct in that they are linked with aforementioned campaigns and WUNC displays.

WUNC displays, according to Tilly and Wood (5), "can take the form of statements, slogans, or labels that imply worthiness, unity, numbers, and commitment." WUNC displays are forms of communication intended to express solidarity within the collective while representing the collective to the public in recognizable fashions. The choice of particular WUNC displays is contingent on the movement's setting, and thus may vary according to public receptiveness. For example, Tilly and Wood (5) lists WUNC displays common to many of the social movements they describe:

- worthiness: sober demeanor; neat clothing; presence of clergy, dignitaries, and mothers with children;

- unity: matching badges, headbands, banners, or costumes; marching in ranks; singing and chanting;

- numbers: headcounts, signatures on petitions, messages from constituents, filling streets;

- commitment: braving bad weather; visible participation by the old and handicapped; resistance to repression; ostentatious sacrifice, subscription, and/or benefaction.

As with Tarrow's conclusion regarding contentious politics in general, Tilly and Wood find that social movements are instigated and shaped by their political and 
economic context. An important implication is that Tilly and Wood (15) also finds that "the social movement, as an invented institution, could disappear or mutate into some quite different form of politics.” In particular, they observe how globalization may be instigating changes to social movements.

\section{The Cypherpunks}

The Cypherpunks originated with a rather innocuous mailing list crafted to pull software developers together worldwide in discussing and solving technological issues. In 1992, the group of San Francisco bay area developers began writing to discuss cryptography, mathematics, and general computer science. Their membership grew to a height of about 2,000 by 1997 , while topics of interest widened to include political matters, specifically regarding state surveillance and control of information on the Internet. Robert Manne (2011) summarizes:

[a]t the core of the Cypherpunk philosophy was the belief that the great question of politics in the age of the Internet was whether the state would strangle individual freedom and privacy through its capacity for electronic surveillance or whether autonomous individuals would eventually undermine and even destroy the state through their deployment of electronic weapons newly at hand.

While the Cypherpunks struggled to gain traction outside their insular community, ultimately dwindling in numbers and activity by the year 2000 , they articulated the political claim to untraceable electronic cash and laid the foundations for the emergence of Satoshi Nakamoto and Bitcoin.

In the early 1990s, tensions between cryptography enthusiasts and US authorities reached a fever pitch. Legislation making its way through the US Congress in 1991 galvanized cryptography enthusiasts who collaborated through online bulletin board 
systems (BBSes). The Comprehensive Counter-Terrorism Act of 1991 buried language that would have permitted the government "to obtain the plain text contents of voice, data, and other communications when appropriately authorized by law." Though the bill failed to pass, Levy $(2001,196)$ explains that within months of its introduction, an NSA contractor familiar with the bill warned members of various BBSes to "begin to stock up on crypto gear while you can still get it." The warning inspired anti-nuclear activist and cryptography enthusiast Phil Zimmerman to freely distribute the encryption software, Pretty Good Privacy (PGP).

Just as consequential, if not more so, the warning inspired what Levy (205) calls "cryptoactivism": using the Internet to promote and distribute strong cryptography. Its original proponents, Tim May and Eric Hughes, were according to Levy (205-206), a pair of intensely libertarian, slightly paranoid crypto enthusiasts. They understood privacy as a fundamental human right, and viewed cryptography as essential to privacy on the Internet. Hughes (in Levy 207) said "it used to be that you could get privacy by going to the physical frontier, where no one would bother you. With the right application of cryptography, you can again move out to the frontier-permanently.” By mid-1992, May and Hughes decided to follow in Zimmerman's footsteps and develop, distribute, and promote tools the public could use to protect their privacy against third parties, namely "cyberthieves, credit bureaus, and especially the government" (207). They recruited fellow libertarian and Electronic Frontier Foundation (EFF) co-founder John Gilmore. At the first "Conference on Computers, Freedom and Privacy," organized by Computer Professionals for Social Responsibility (CPSR), Gilmore had recently delivered a talk entitled "Privacy, Technology, and the Open Society." He imagines: 
What if we could build a society where the information was never collected? Where you could pay to rent a video without leaving a credit card number or a bank number? Where you could prove you're certified to drive without ever giving your name? Where you could send and receive messages without revealing your physical location, like an electronic post office box?

That's the kind of society I want to build. I want a guarantee-with physics and mathematics, not with laws - that we can give ourselves things like real privacy of personal communications. Encryption strong enough that even the NSA can't break it. We already know how. But we're not applying it. (Gilmore 1991)

Exasperated by that last phrase, "we're not applying it," May, Hughes, and Gilmore organized an invitation-only group of like minds to figure out how to get strong crypto in the hands of the public. Mimicking the academic CPSR, Hughes initially called the group "Cryptology Amateurs for Social Irresponsibility" (CASI). About twenty attended its first, late summer meeting in Berkeley. Levy $(2001,209)$ describes them as a mix of libertarians and "more than a few [...] self-proclaimed Extropians, whose philosophy merged an extremist view of individual liberties with a loopy belief that the far fringes of scientific research would soon accrue to our benefit." One of them, Jude Milhon styled the group "Cypherpunks," a play on contemporary cyberpunks, "hackers turned hipsters by linking the in-your-face iconoclasm of punk-rock rebels with the digital revolution" (Levy 211). The name stuck and the Cypherpunks were born.

Stifled by the physical limits of meeting in person, Cypherpunks soon moved into their more natural environment, the Internet, where they assumed a distinct ethos, organized their efforts, and multiplied. On September 21, 1992, Hughes set up the "Cypherpunks mailing list," an online listserv hosted on Gilmore's server whereby Cypherpunks could reach all list members at once. Two weeks later, Hughes posted a 
draft statement of purpose for the group's comment. It ultimately became "A

Cypherpunk's Manifesto," which reads:

Privacy is necessary for an open society in the electronic age. [...]

Cypherpunks write code. [...] We publish our code so that our fellow Cypherpunks may practice and play with it. Our code is free for all to use, worldwide. [...] We know that software can't be destroyed and that a widely dispersed system can't be shut down.

Cypherpunks deplore regulations on cryptography, for encryption is fundamentally a private act. [...] Even laws against cryptography reach only so far as a nation's border and the arm of its violence. Cryptography will ineluctably spread over the whole globe, and with it the anonymous transactions systems that it makes possible.

For privacy to be widespread it must be part of a social contract. People must come and together deploy these systems for the common good. Privacy only extends so far as the cooperation of one's fellows in society. [...]

The Cypherpunks are actively engaged in making the networks safer for privacy. (Hughes 1993)

The Cypherpunk Manifesto followed a more grandiose and pointed "The Crypto

Anarchist Manifesto," read by Tim May at gatherings of crypto enthusiasts including Crypto ' 88 and the Hackers Conference of the same year. May read it again at the first

Cypherpunk meeting:

A specter is haunting the modern world, the specter of crypto anarchy.

Computer technology is on the verge of providing the ability for individuals and groups to communicate and interact with each other in a totally anonymous manner. [...] Interactions over networks will be untraceable $[\ldots]$ Reputations will be of central importance [...] These developments will alter completely the nature of government regulation, the ability to tax and control economic interactions, the ability to keep information secret, and will even alter the nature of trust and reputation.

The technology for this revolution - and it surely will be both a social and economic revolution - has existed in theory for the past decade. The methods are based upon public-key encryption, zero-knowledge interactive proof systems, and various software protocols for interaction, authentication, and verification. The focus has until now been on academic conferences in Europe and the US, conferences monitored closely by the National Security Agency. But only recently have computer networks and personal computers attained sufficient speed to make the ideas practically realizable. And the next ten years will bring 
enough additional speed to make the ideas economically feasible and essentially unstoppable. [...]

The State will of course try to slow or halt the spread of this technology, citing national security concerns, use of the technology by drug dealers and tax evaders, and fears of societal disintegration. Many of these concerns will be valid; crypto anarchy will allow national secrets to be trade [sic] freely and will allow illicit and stolen materials to be traded. An anonymous computerized market will even make possible abhorrent markets for assassinations and extortion. Various criminal and foreign elements will be active users of CryptoNet. But this will not halt the spread of crypto anarchy.

Just as the technology of printing altered and reduced the power of medieval guilds and the social power structure, so too will cryptologic methods fundamentally alter the nature of corporations and of government interference in economic transactions. Combined with emerging information markets, crypto anarchy will create a liquid market for any and all material which can be put into words and pictures. And just as a seemingly minor invention like barbed wire made possible the fencing-off of vast ranches and farms, thus altering forever the concepts of land and property rights in the frontier West, so too will the seemingly minor discovery out of an arcane branch of mathematics come to be the wire clippers which dismantle the barbed wire around intellectual property.

Arise, you have nothing to lose but your barbed wire fences! (May 1992)

Some Cypherpunks took the call to extremes, capturing the attention of federal agencies. For example, Jim Bell, whom Wired correspondent Declan McCullagh (2001) calls "one of the Internet's most famous essayists," introduced the concept of an online funding for the assassination of public figures if they made enough people unhappy. In a 1995 series of posts to the "outpost-of-freedom.com," titled "Assassination Politics," Bell writes how electronic cash in particular makes such an assassination pool possible anonymously. Within two years, Bell ended up in prison for the better part of the next 15 years on charges ranging from violating probation, harassing a federal agent, and evading taxes.

While Bell took the application of electronic cash to an extreme, discussions of electronic cash had been a feature of the Cypherpunks since the group's beginning. In an estimated 37,633 posts to the mailing list between 1992 and 1997, members mentioned 
electronic cash 9,957 times. ${ }^{16}$ Cypherpunk discussions of electronic cash typically varied along technical lines, but generally coalesced around the interests described in Hughes's “A Cypherpunk’s Manifesto”:

$[\ldots]$ privacy in an open society requires anonymous transaction systems. Until now, cash has been the primary such system. An anonymous transaction system is not a secret transaction system. An anonymous system empowers individuals to reveal their identity when desired and only when desired; this is the essence of privacy. [...]

We the Cypherpunks are dedicated to building anonymous systems. We are defending our privacy with cryptography, with anonymous mail forwarding systems, with digital signatures, and with electronic money. (Hughes 1993)

While the Cypherpunk mailing list inspired a relatively small number of crypto enthusiasts, it had limited immediate impact, little public recognition, and struggled to endure. Only a year after the group's formation, some members began to worry about the community stalling. The group's numbers were up, but growth brought new tensions, particularly as posts gravitated toward politics and featured the founding firebrands Gilmore, Hughes, and May. Not everyone who joined, it turned out, was a libertariancrypto-anarchist. In August 1993, Nick Szabo attempted unifying the group by reminding members that they were responsible for PGP and anonymous remailers, ${ }^{17}$ and that they would play a large role in defeating the NSA's Clipper Chip. ${ }^{18}$ Szabo implored them to downplay divisions and to focus on "practical stuff," suggesting that working on projects like "digital cash" would unify its membership. Ultimately, a row within its ranks over

\footnotetext{
${ }^{16}$ Author's estimates. The number of posts are derived from instances of "To: cypherpunks@toad.com" and the number of mentions of electronic cash are derived from instances of "digital cash," "digicash," "electronic cash," "digital money," "electronic money," and "e-money." Data is from the "Cypherpunks Mailing List Archive," http://mailing-list-archive.cryptoanarchy.wiki/.

${ }^{17}$ Anonymous remailers are servers that enable email communication without disclosing identities. ${ }^{18}$ A project of the NSA unveiled in 1993, the Clipper Chip was an encryption device with a "backdoor" that enabled law enforcement to decode encrypted transmissions. After public backlash, the Clinton Administration abandoned the project by 1996.
} 
whether or not the group should become moderated—somewhat ironically for a group opposed to the control of information-overshadowed any attempt at unifying the community. By February 1997, the mailing list split in two: one moderated and one "distributed." With that, the Cypherpunks receded.

\section{Bitcoin}

As in Chapter 2's analysis of Bitcoin as an actor in global affairs, this section considers Bitcoin's social network as a transnational actor, a "[form] of coordinated or collective action aimed at changing international outcomes and national policies" (Kahler 2009, 5). The Bitcoin social network comprises interdependent actors (or "nodes" in the nomenclature of network analysis) — millions of users as well as hundreds of service providers worldwide — connected by information flows (or "links"). This section builds on Chapter 2's description of Bitcoin's social network to facilitate the following section's discussion of Bitcoin as a social movement.

Bitcoin's social network emerged from a political and economic context characterized by surveillance, globalization, and economic crisis. As with the Cypherpunks, surveillance — specifically that conducted by US security agencies—was a prevailing concern of those behind Bitcoin. During the decade leading up to Bitcoin's release, they had ample evidence that surveillance had not much abated. Over the same period, globalization accelerated, particularly in the form of increasing Internet access, ecommerce volumes, and digitization. Finally, Bitcoin's release in January 2009 occurred during the depths of the Great Recession. Whether or not the timing was intentional, it had implications for Bitcoin's salience in a crisis environment. 
Signed into law on October 26, 2001, the USA PATRIOT Act greatly expanded the US federal government's authority to gather domestic intelligence on national security grounds. The PATRIOT Act granted the NSA and FBI unprecedented surveillance powers, ratcheting up crypto enthusiasts' efforts to secure data and communications while agitating the likes of advocacy organizations including the EFF, the Electronic Privacy Information Center (EPIC), and the Center for Democracy and Technology (CDT). Unsurprisingly, Bitcoin found its home in these circles, as they were devoted to cryptographic protections from state surveillance. In 2008, Nakamoto posted the Bitcoin white paper to the Cryptography Mailing List, a moderated descendent of the original Cypherpunks Mailing List. Established in 2001, the Cryptography Mailing List is "devoted to cryptographic technology and its political impact [...including...] social repercussions of cryptosystems, and the politics of cryptography such as export controls or laws restricting cryptography" (cryptography-owner@metzdowd.com 2020). List members quickly took up Bitcoin's technical, economic, and political implications, setting out to get the electronic cash off the ground.

Operations conducted under PATRIOT Act authorization also fueled the rise of WikiLeaks. Launched in 2006, WikiLeaks uses strong encryption to gather material while maintaining the anonymity of its sources. It then publishes the oft-classified material on its website, usually incriminating states and individuals in conducting illegal or unethical activities. One of WikiLeaks's most influential releases, beginning in June 2013, disclosed an unprecedented scale of global surveillance conducted in large part by US authorities. The exposure provoked widespread uproar among digital privacy advocates, especially among those in the cryptography community. Furthermore, 
coverage of the leaks in the popular press appears to have raised public interest in the somewhat obscure realm of cryptography. Some businesses, such as Apple and Google, responded with stronger encryption and privacy claims, while Bitcoin saw its first surge in popular interest. While it is difficult to isolate WikiLeak's effect on public interest in Bitcoin from that of Mt. Gox's and Silk Road's public notoriety during 2013-14, according to Google Trends (2020) "Interest over time" for the search term "bitcoin," the twelve months immediately following the surveillance leaks reflect its greatest annual search volume between January 2009 and July 2017.

Globalization has also played a significant role in Bitcoin's development. As it reflects a world increasingly connected via the Internet and dependent on e-commerce and digitization, globalization makes Bitcoin possible. Tilly and Wood $(2012,99)$ describes globalization as a process in which "a distinctive set of social connections and practices expands from a regional to a transcontinental scale," outpaces the countervailing process in which "an existing set of transcontinental social connections and practices fragments, disintegrates, or vanishes." Between the mid-1990s and the time of Bitcoin's release, the "set of transcontinental social connections and practices" with respect to the Internet and e-commerce dramatically intensified. In 1995, there were approximately 44 million Internet users. By 2000, the number of Internet users grew nearly tenfold to about 412 million, and in 2017, included almost half the world population, over 3.7 billion (World Bank 2020). Over roughly the same period, ecommerce - transactions ordered over the Internet for goods and services that "may be delivered either digitally or physically" (WTO 2018, 8) — grew astronomically. In 1995, e-commerce was a negligible share of the global economy. While precise measures are 
difficult to establish for the early days of e-commerce, it is illustrative to note that Amazon had been less than a year old and eBay was just launched at the time (as was the World Trade Organization). The WTO $(2018,5)$ estimates that global e-commerce, both domestic and cross-border, had grown to $\$ 27.7$ trillion by 2016 , over a third of world GDP. ${ }^{19}$ The Internet's spread and the growth of e-commerce have proceeded in tandem with digitization, converting analog information into digital information so computers can process and transmit it. To summarize, Bitcoin would be limited had transactions not increasingly moved online since the days of the Cypherpunks. Indeed, the 2018 WTO report confirms that blockchain technology more broadly "ha[s] been made possible by these same forces" (6).

Changing economic conditions-often, but not always linked with globalization - have also contributed to an environment receptive to Bitcoin. The Great Recession's depressive effect on individual trust in public institutions may make Bitcoin an appealing alternative to such institutions. Examining the results of the General Social Survey and the Gallup World Poll, Betsey Stevenson and Justin Wolfers (2011) finds that respondents' trust in government and financial institutions ${ }^{20}$ declined precipitously in most countries since the Great Recession. The US public's declining trust in banks was particularly sharp, falling from over $50 \%$ of respondents expressing "a great deal" or

\footnotetext{
${ }^{19}$ It is difficult to distinguish the shares of domestic and cross-border e-commerce because the data from which these estimates are derived does not identify origin. However, $\operatorname{UNCTAD}(2017,32)$ estimates that cross-border online business to consumer purchases amounted to about $\$ 189$ billion in 2015 , about $1.4 \%$ of total merchandise imports.

${ }^{20}$ GSS respondents are asked the question, "Now I am going to read you a list of institutions in American society. Please tell me how much confidence you, yourself, have in each one." Available responses are arranged in a 4 point Likert scale. Gallup World Poll respondents are asked, "In [country], do you have confidence in each of the following, or not?" Institutions listed include the "national government" and "financial institutions or banks." An affirmative answer is coded as " 1 " and all other answers, including don't know or refused, are coded as " 0. ."
} 
"quite a lot" of confidence in banks during 2006 to less than 25\% by 2010. Alan S.

Blinder $(2013,26)$ further probes the Great Recession and trust in government, revealing a "macroeconomic policy paradox." Blinder (27) finds that interventions by the US Congress and Federal Reserve were successful in stemming the worst of the Recession, but led to "a backlash against the Federal Reserve; against the Obama administration, Congress, and especially Democrats; against Keynesian economics; and most certainly against policy activism." Aggressively loose monetary policy, for example, led "the public to judg[e] the Fed to have done the worst job on a list of nine federal agencies that included Homeland Security, the CIA, and even the IRS" (37-38). As an autonomous electronic cash system with a predetermined money supply, the Great Recession and its aftermath may have catalyzed interest in Bitcoin as an alternative to institutions rapidly losing trust, as well as increasing its appeal among those wary of monetary intervention.

\section{From Contention to A Digital Social Movement}

The evidence indicates there is much in common between the Cypherpunks and Bitcoin's social network. ${ }^{21}$ Both meet Tarrow's $(2013,266)$ definition of contentious politics and operate outside, but intersect with "routine political processes-like elections and interest group politics." In the case of the Cypherpunks, the mailing list members were claimants who made claims ranging from Internet privacy to "crypto anarchy." State surveillance agencies such as the NSA, regulators, and their corporate counterparts - especially AT\&T (having partnered with the NSA on the Clipper Chip project) — were the objects of the Cypherpunks' claims in that they were to cease

\footnotetext{
${ }^{21}$ See Table 2. Summary of Findings: The Cypherpunks and Bitcoin as Contentious Politics.
} 
surveillance activities. Similarly, using Bitcoin represents a claim to Internet privacy and the freedom of peer-to-peer transaction. Monetary authorities and incumbent financial institutions become the objects of Bitcoin's claims, for they are to withdraw from among users' digital transactions. Both the Cypherpunks and Bitcoin have employed repertoires of collective direct action to contest government surveillance, ranging from developing and freely distributing cutting-edge encryption technologies to, of course, untraceable electronic cash.

The similarities between the Cypherpunks and Bitcoin break down, however, when evaluating whether or not they constitute social movements. According to Tilly and Wood's three core elements of social movements, the Cypherpunks fell short of a movement whereas Bitcoin resembles a social movement. The differing outcomes are broadly indicative of the Cypherpunks' insular character, failure to represent themselves to the public in recognizable fashions, and lack of crosscutting coalition building; by contrast, Bitcoin has become open, its recognizable, and able to generate crosscutting coalitions.

Bitcoin has, to use Tilly and Wood's $(2012,12)$ expression, “proceeded not as [a] solo [performance], but as [an] interactive [campaign].” That is, Bitcoin involves sustained, organized effort linking the millions of Bitcoin users with the wider public, in opposition to monetary authorities and incumbent financial institutions. As described below, "Worthiness, Unity, Numbers, and Commitment" (WUNC) displays link Bitcoin users with the public, while Bitcoin's repertoire of contention characterizes the interactions between its users as claimants of untraceable electronic cash and third parties including allies, rival cryptocurrencies, incumbent financial services, technology firms, 
authorities, and the public. Bitcoin also involves what Tilly and Wood (13) calls “program, identity, and standing” claims. Bitcoin's program claims include opposition to incumbent financial services, monetary authorities, and surveillance of all forms. Its identity claim, reinforced by WUNC displays, asserts that Bitcoin users are an influential collective. Bitcoin's standing claims are twofold: first, that Bitcoin is tied to other political actors such as online privacy advocates, financial inclusion activists, and local opposition groups; second, that it calls for the exclusion of incumbent financial services firms and state authorities from electronic transactions.

Another observation Tilly and Wood (13) makes is that "the relative salience of program, identity, and standing claims varies significantly [...] among claimants within social movements, and among phases of social movements." Chapter 2 extensively presents this dynamic in Bitcoin, finding that Bitcoin's economic imperatives have undermined its collective political aims. However, as the present chapter suggests, Bitcoin's history is far from over. It may be that if Bitcoin exhibits greater success as a form of money_-more widespread use, pricing in bitcoin, and lower price volatility—its political claims will once again take center stage. Recall, too, that for a substantial share of (illicit) Bitcoin activity, its claims to Internet privacy and peer-to-peer transaction appear to have been realized. Another example of Bitcoin's variation in the salience of claims relates to different local contexts. Returning to the Google Trends (2020) "Interest over time" search for the term "bitcoin," it is noteworthy that between January 2009 and May 2020, the most common search origin was Nigeria (indexed at 100), followed by South Africa (indexed at 71), and Ghana (indexed at 66). By comparison, searches for "bitcoin" originating in the US are fourteenth on the list, indexed at 33. As home to a 
disproportionate share of worldwide digital crime such as hacking and phishing (Odiboh et al. 2017), Nigeria may be a natural source of interest in Bitcoin. With Ghana and South Africa, Nigeria shares two additional features that may foster interest in Bitcoin: an increasingly dominant share of payments occurring over mobile phones (to which Bitcoin is particularly well-suited), as well as exchange rates that make using bitcoin favorable to using the Ghanaian cedi, South African rand, and Nigerian naira. Ultimately, variations of claim salience over time and across the global Bitcoin network make Bitcoin appear more like the social movements Tilly and Wood describe than not.

The Bitcoin social network's repertoire of contention centers around direct cyber action outside routine political processes and institutions, including a substitution boycott; a quasi-clandestine, networked form of organization; and crosscutting coalition building. First, using bitcoin is a form a substitution boycott in that it simultaneously represents a withdrawal from conventional currency use and a substitution for that use with bitcoin. Observers may object that the use of bitcoin may not be politically motivated and thus not constitute a form of protest. Admittedly, Bitcoin serves a dualpurpose in this respect: some users may be drawn to Bitcoin for its political implications, while other users may be drawn to its speculative, technological, or simple functional appeal. However, the boycott effect of using bitcoin, when it replaces the use of conventional currencies remains the same regardless of its user's motivations. Second, Bitcoin's quasi-clandestine, networked form of organization cannot be overstated. As a transnational network with no formal authority, it presents no single point of failure while straddling numerous jurisdictions, making the network resilient to attack. Those who choose to participate pseudonymously, moreover, are afforded even greater protection 
from surveillance, censorship, and enforcement. Finally, because Bitcoin appeals to groups interested in more than just Internet privacy or peer-to-peer transaction, it reflects crosscutting coalition building online. Bitcoin advocates now include those promoting financial inclusion, consumers, investors, disruptive financial technology enterprises, and illicit networks.

Bitcoin's WUNC displays, while unconventional, are still recognizable as methods of linking Bitcoin users with the public. Bitcoin is presented as an alternative form of trust— - based on cryptography, computer code, and networked organizationrather than on human institutions. In an environment where traditional institutions inspire historically low levels of public trust, the alternative form of trust is Bitcoin's claim to worthiness. Similarly, Bitcoin displays unity largely through technological means: uniform wallet addresses placed on website as well as QR codes representing sources and destinations for bitcoin. Most recognizably, the bitcoin symbol, $\square$, has been part of the Unicode text standard since June 2017 and is featured on widespread illustrations of a gold-colored coin (a physical facsimile of its digital reality). While bitcoin is exclusively digital, this physical representation is likely widely recognizable to the public. Bitcoin also features public displays of its numbers: from physical conferences and social media swarming by Bitcoin enthusiasts, to staggering volumes (460 million Bitcoin addresses as of December 2018) (Tassev 2018) and market prices (1 bitcoin trades for about $\$ 9,700$ as of May 14, 2020) (CoinMarketCap 2020). Still, given wide fluctuations in the price of bitcoin (approaching $\$ 20,000$ in December 2017 and descending to nearly $\$ 3,000$ one year later), those willing to brave wild market swings display commitment. Perhaps even 
more noteworthy is the commitment displayed by users in countries where Bitcoin is outlawed, or even those who take the risk of it being seized, stolen, or lost.

Table 2. Summary of Findings: The Cypherpunks and Bitcoin as Contentious Politics

\begin{tabular}{|c|c|c|c|}
\hline & & The Cypherpunks & Bitcoin \\
\hline \multirow[t]{4}{*}{$\begin{array}{l}\text { Contentious } \\
\text { Politics }\end{array}$} & Claims & $\begin{array}{l}\text { Ranging from Internet privacy to } \\
\text { "crypto anarchy" }\end{array}$ & $\begin{array}{l}\text { Internet privacy and freedom of } \\
\text { peer-to-peer transaction }\end{array}$ \\
\hline & Claimant & Cypherpunk mailing list members & The Bitcoin social network \\
\hline & $\begin{array}{l}\text { Objects of } \\
\text { claims }\end{array}$ & $\begin{array}{l}\text { State surveillance agencies, } \\
\text { regulators, and their corporate } \\
\text { counterparts }\end{array}$ & $\begin{array}{l}\text { Monetary authorities and } \\
\text { incumbent financial institutions }\end{array}$ \\
\hline & $\begin{array}{l}\text { Character of } \\
\text { interaction }\end{array}$ & Episodic, public, and collective & Episodic, public, and collective \\
\hline \multirow[t]{3}{*}{$\begin{array}{l}\text { Social } \\
\text { Movement }\end{array}$} & Campaign & $\begin{array}{l}\text { Sustained, organized effort that did } \\
\text { not adequately link Cypherpunks } \\
\text { and the Internet-using public, } \\
\text { contra state actors and corporate } \\
\text { counterparts }\end{array}$ & $\begin{array}{l}\text { Sustained, organized effort linking } \\
\text { Bitcoin users with the public, } \\
\text { contra monetary authorities and } \\
\text { incumbent financial institutions }\end{array}$ \\
\hline & Repertoire & $\begin{array}{l}\text { Direct cyber action outside political } \\
\text { institutions: open source coding, } \\
\text { hacktivism; networked form of } \\
\text { organization; lack of crosscutting } \\
\text { coalition building }\end{array}$ & $\begin{array}{l}\text { Direct cyber action outside political } \\
\text { institutions: boycott, quasi- } \\
\text { clandestine networked form of } \\
\text { organization; crosscutting coalition } \\
\text { building (financial inclusion, } \\
\text { consumers, illicit networks, } \\
\text { investors, disruptive FinTech } \\
\text { enterprises) }\end{array}$ \\
\hline & $\begin{array}{l}\text { WUNC } \\
\text { displays }\end{array}$ & $\begin{array}{l}\text { Worthiness: insufficiently } \\
\text { recognizable to public at large } \\
\text { Unity: not achieved } \\
\text { Numbers: imperceptible to public } \\
\text { Commitment: defying law }\end{array}$ & $\begin{array}{l}\text { Worthiness: presented as } \\
\text { alternative form of trust } \\
\text { Unity: symbols, wallet addresses, } \\
\text { QR codes } \\
\text { Numbers: conferences, wallets, } \\
\text { bitcoin performance, social } \\
\text { media swarming } \\
\text { Commitment: braving market } \\
\text { swings, defying repressive } \\
\text { regimes, risk-taking }\end{array}$ \\
\hline
\end{tabular}

Whereas the Cypherpunks' repertoire of contention—-based on direct cyber action (such as open source development of encryption tools and what we now call "hacktivism") and networked organization—shares elements with that of Bitcoin, unlike Bitcoin, the Cypherpunks failed to successfully engage with the public and build crosscutting coalitions. Furthermore, WUNC displays by the Cypherpunks were either 
not evident or unlikely to be recognized favorably by the public. Figure 6 exhibits Bitcoin taking the instantly recognizable, perhaps innocuous form of an ATM. ${ }^{22}$ Ultimately, while both the Cypherpunks and Bitcoin reflect contentious politics, only Bitcoin meets Tilly and Wood's definition of a social movement.

Figure 6. Bitcoin Kiosk (or "ATM")

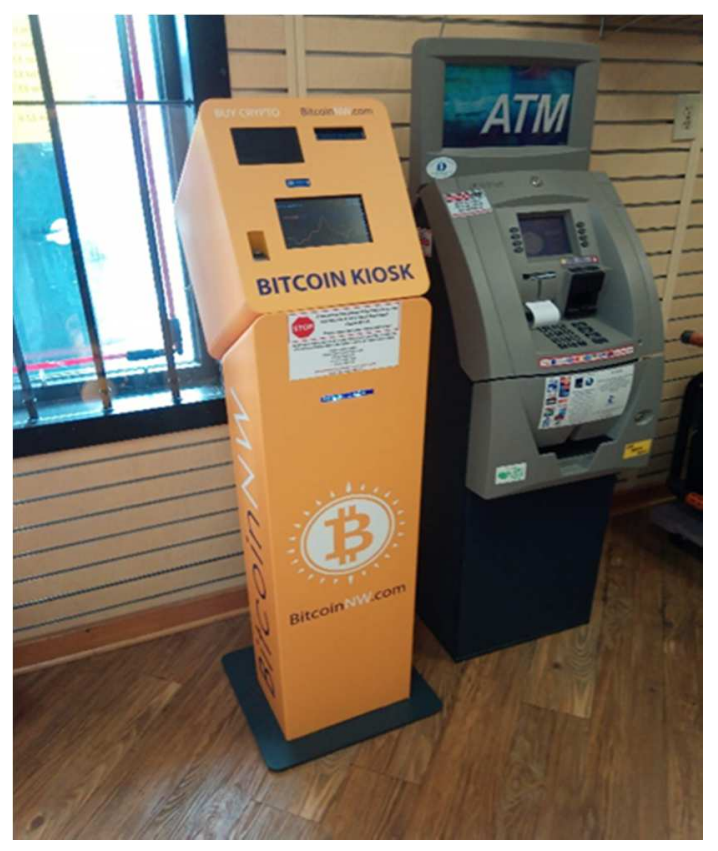

Source: Will Baker (2020).

The difference between the Cypherpunks' failure to survive and Bitcoin's success stems in large part from their different responses to particular political and economic contexts. The Cypherpunks chose to pursue a relatively insular strategy of direct action against the US government in particular, neglecting to build public recognition and

\footnotetext{
${ }^{22}$ To view the contrast between this image of Bitcoin and the Cypherpunks' curious-if not eerie-public image, see Larry Dyer's 1993 Wired magazine cover photograph at https://www.wired.com/story/wiredcover-browser-1993/.
} 
support. While Bitcoin's political and economic context also galvanized its early supporters to pursue direct action, the preceding analysis suggests its specific repertoire and recognizable WUNC displays have increased Bitcoin's salience to the wider public.

\section{How Bitcoin and Blockchain are "Shaking the System"}

Many implications of Bitcoin and blockchain are conditional. If Bitcoin adoption grows to some critical mass and if applications of blockchain technology bear fruit beyond untraceable electronic cash, then impacts become more tangible. That said, this thesis shows that Bitcoin has already succeeded in several respects: transnational cyber action (at least with respect to illicit activities), the provision of blockchain as a public good, and achieving the status of a social movement. Together, these successes suggest Bitcoin and blockchain will persist and merit further inquiry into their implications. Possible topics of interest to scholars of international relations and international political economy include impacts on international capital markets; foreign exchange reserves and currency competition; central bank monetary policy autonomy; crime, terrorism, and sanctions; and even systems of trust. Indeed, these are likely the topics Lagarde had in mind when commenting that blockchain technology is "shaking the system."

\section{International Capital Markets}

As a globally exchanged asset that remains largely linked with official currencies, bitcoin may shed light on significant developments in capital markets. For example, Gina Pieters (2016a, 31-32) uses bitcoin price data to measure unofficial exchange rates and to 
evaluate international capital flows with respect to national capital controls. Pieters (31) concludes that:

Bitcoin-based exchange rates can be used to identify episodes of capital movement that, due to their transitory nature, current classification systems [...] cannot detect. This method of identification has powerful implications for future applied and policy work, as bitcoin data are publicly available at no charge on a daily basis - even as events unfold - and cannot be manipulated by bad reporting as could be the case with the World Currency Yearbook or, to a lesser extent, the IMF classification system. Additionally, even if governments temporarily cease to gather data due to political or economic upset, the bitcoin data continue to exist and accrue.

Pieters's analysis shows how bitcoin data may be used to more accurately detect changes in global financial markets than before, but also points to how Bitcoin's use poses a challenge to countries attempting to impose capital controls, such as Argentina, China, or Russia. Pieters and Sofia Vivanco (2016, in Pieters 2016b, 25) find that "government attempts to regulate the globally accessible bitcoin markets are generally unsuccessful and $[\ldots]$ bitcoin exchange rates tend to reflect the market, not official exchange rates."

\section{Foreign Exchange Reserves and Currency Competition}

At the end of 2019, the US dollar accounted for $57 \%$ of foreign exchange reserves worldwide, followed by the euro at 19\% (IMF 2020). US dollar dominance in foreign exchange reserves has long been understood as a source of US power. Benjamin Cohen $(2008,457)$ summarizes that "the issuers of currencies that are widely used by others as reserve assets $[\ldots]$ can finance deficits simply by printing more of their own money." This ability to finance deficits grants the US unparalleled international monetary autonomy, which increases its potential leverage vis-à-vis the rest of the world. Andrea 
O’Sullivan $(2017,95-96)$ entertains the possibility that "Bitcoin may, in the long-run, replace the US dollar as the world's reserve currency." While the suggestion seems farfetched, O'Sullivan roots Bitcoin in the tradition of Friedrich Hayek, arguing that it "may just be a 'sly roundabout way' to force reform of the global monetary system." At present, however, there is little evidence to suggest that countries are adding Bitcoin to their mix of reserves.

Another avenue to consider is that China's digitized renminbi is an attempt to shift foreign reserves toward the renminbi. Less than $2 \%$ of worldwide foreign exchange reserves are held in renminbi. A Chinese digital currency would be exceptional given the country's geostrategic position and ambitions. John Hemmings (2020) situates a Chinese digital currency squarely within its Digital Silk Road, a deliberate strategy to project power 'through technology 'bundles' comprised of smart cities, smart ports, e-commerce [...], communications networks, and satellite networks" (7). Ultimately, Hemmings argues that China's digital renminbi is intended to "lessen the utility of the dollar while simultaneously increasing Beijing's oversight of all financial activity [across the Digital Silk Road]" (11-12). There are indications that Chinese leadership are widely embracing the project. The "Digital Currency Electronic Payment" system, which President Xi Jinping has been increasingly touting, is under development by several state banks and state-owned telecom companies. Covering an October 2019 Politburo meeting, Xinhua (2019) quotes President Xi remarking on digital currency and blockchain, "it is essential for blockchain technology to play a bigger role in building China's strength in cyberspace, developing the digital economy and advancing the economic and social development.” The same month, China's People’s Daily Press published 数字货币一领导 
干部读本 (Digital Currency: A Reader for Cadres), a 200 page guide to digital currency basics, monetary systems, and global finance. It was a surprise best-seller, having entered a second printing within months (Zhao 2020).

\section{Central Bank Monetary Policy Autonomy}

According to Pieters and Vivanco (2016, in Pieters 2016b, 25), if Bitcoin facilitates sufficiently large enough capital flows, "all countries will have, by default, unrestricted international capital markets." The result would force countries to choose between managing their exchange rates through monetary policy or pursuing monetary independence through floating exchange rates.

\section{Crime, Terrorism, and Sanctions Avoidance}

Three major categories of illicit Bitcoin use have been developed to date: criminal activity, terrorism finance, and sanctions avoidance. Recall that Foley, Karlsen, and Putniņš $(2019,1798)$ estimates $46 \%$ of bitcoin transactions each year are used in criminal activity. Online security firm Coveware (2020) reports that Bitcoin is now the preferred method of payment in ransomware, accounting for $99 \%$ of attempted cyber extortions. While there is limited evidence of Bitcoin's use in terrorism finance to date, DionSchwarz, Manheim, and Johnston (2019) finds that it may be only a matter of time, depending largely on future development of the network. Luke McNamara (2017), of the cybersecurity firm FireEye, concludes that North Korea has increasingly turned to using bitcoin in an effort to evade US sanctions, while others (Ratna 2020; Krygier 2018) suggest Iran and Venezuela have followed suit. According to the United Nations Security 
Council, 1718 Sanctions Committee (DPRK) Panel of Experts $(2019,49)$ "cyberspace is used by the DPRK as an asymmetric means to carry out illicit and undercover operations in the field of cybercrime and sanctions evasion. These operations aim to acquire funds through a variety of measures in order to circumvent the sanctions." The same report (51) concludes that "the Democratic People's Republic of Korea carried out at least five successful attacks against cryptocurrency exchanges in Asia between January 2017 and September 2018, resulting in a total loss of $\$ 571$ million." The total theft amounts to about 20\% of North Korea's estimated \$3 billion cash reserves as of February 2018 (Harris 2018).

\section{Systems of Trust}

Perhaps unsurprisingly, a common theme among Bitcoin observers is how Bitcoin represents an alternative to incumbent institutions, rules, and norms. All the same, to call Bitcoin disorderly or anarchic would be folly. Indeed, Bitcoin activities including communication, transactions, and mining, each follow observable, rule-based patterns. Governance takes an entirely new form with Bitcoin, suggesting what Kevin Werbach (2018) describes as a "new architecture of trust." This new, blockchain trust architecture - in contrast to interpersonal trust, intermediary trust, and state-enforced trust — displaces the need to trust any individual component of a system of agreements toward trust in the system as a whole. For Werbach, "cryptocurrency networks are technologies of governance. The blockchain decentralizes the rule-enforcement aspects, but not necessarily rule creation" (134). That is, behaviors in Bitcoin—specifically communications, transactions, and mining - are constrained by the Bitcoin protocol. It is 
computationally impractical, if not impossible, to break those rules. ${ }^{23}$ The issue Werbach suggests, then, is how changes to the protocol are made and who has influence over such changes. Werbach concludes that, because blockchain does not eliminate the need for trust, the technology must be situated in a wider, adaptive legal framework.

${ }^{23}$ Distinguishing between impractical and impossible grants that, in some cases, code may become outmoded by advances in technology, such as quantum computing, or subverted by malicious attacks, such as hacking. 


\section{Chapter 5: Conclusion}

While the implications of Bitcoin and blockchain technology remain largely speculative, this thesis shows that political scientists should not overlook Bitcoin and blockchain technology. Bitcoin operates like a political network and has achieved observable collective success in global affairs. Bitcoin's application of blockchain technology is a novel example of public goods provisioning. As a social movement, Bitcoin reflects state-society conflict over technology and illuminates our understanding of privacy and transaction in cyberspace. Finally, Bitcoin presents a way to sidestep political and economic institutions. Each of these reasons to study Bitcoin and blockchain should be all the more compelling as human action and interaction are increasingly conducted in cyberspace. In concluding this thesis, I summarize the key findings of each of the preceding chapters, making note of why they matter to political scientists and what political scientists may contribute to the growing body of literature on Bitcoin and blockchain technology.

Chapter 2 describes Bitcoin as a technical innovation and the first peer-to-peer, untraceable electronic cash. The analysis of Bitcoin as an actor in global affairs shows how Bitcoin has had limited success but fallen short of its overarching political purpose. Again, it is not a failure of the technology per se, but a result of Bitcoin's course of development as a social network. For the most part, the network's attempt to subvert state authority via untraceable electronic cash fell victim to the economic imperatives of its increasingly influential service providers. States appear to be capitalizing on this vulnerability, coordinating enforcement action through KYC and AML/CFT regimes. 
Further analysis might consider how Bitcoin may inspire international cooperation through efforts such as the Automated Exchange of Information, an anti-tax evasion framework promulgated by the OECD. Still, Bitcoin's development reflects a politicaleconomic paradox, not a contradiction. While Bitcoin's economic imperative has outstripped its overall political purpose so far, this is not to say that it will fail to achieve its political purpose. In fact, it is entirely possible that, in order to attain its political purpose, it must first achieve more widespread use. Bitcoin's increasing economic viability may just be the path to such adoption.

Chapter 3 discusses blockchain technology, conceptualizes blockchain as a public good, and analyzes Bitcoin as a public goods provider. As a public goods provider, Bitcoin has fared remarkably well given its large, voluntary membership. In fact, it has done so well at harnessing processing power from users around the world that, by at least one measure, it is the world's most powerful computing network. The predicted effects of combining selective incentives with a threshold may also extend to other forms of networked-provisioned public goods, an increasingly relevant feature of human activity in cyberspace. It is also worth considering how blockchain technology may facilitate the exchange of information among mutually distrustful counterparties, particularly in the international domain. Consider information as a public good, such as that involved in monitoring the spread of a public health pandemic. Authorities have strong incentives to find out about transmission rates and vectors across jurisdictional boundaries, but may be reluctant to identify the infection state of any particular individual or to divulge information to a third party intermediary. If the incentives were sufficient, a blockchain 
solution could enable de-identified information sharing among a network of such authorities.

It is also worth examining blockchain's potential in human development. Some Bitcoin supporters - and for that matter, those of Facebook's planned electronic cash, Libra, or the thousands of altcoins - endorse the technology as a way to empower the estimated 1.7 billion "unbanked" people worldwide (Demirgüç-Kunt et al. 2018, 4). For example, the Libra white paper (Libra Association 2019, 3) describes its purpose as becoming "a simple global currency and financial infrastructure that empowers billions of people." But we must inquire into this claim critically. For one, it implies that the unbanked remain so for lack of access to financial services. However, Demirgüç-Kunt et al. (39-40) find that most of the unbanked cite lack of money as the primary reason they do not have financial accounts. Second, given the information blockchain retains about its users, it is worth scrutinizing how development outcomes might come at the price of monetizing user data. Libra is specifically subject to such scrutiny given its potential embeddedness in a vast network of Facebook platforms with 2.6 billion monthly active users (Facebook 2020).

Chapter 4 argues that Bitcoin matters because it has assumed the form of a resilient, networked social movement adapted to the twenty-first century. This is perhaps one of the most striking feats of Bitcoin: clandestinely assuming an important function of the state, without presenting a single point of failure that would make the network vulnerable to attack. To be sure, alternative currency arrangements are not a novel phenomenon. However, none has achieved the scale and efficiency of Bitcoin, with such rapidity. Whether or not Bitcoin and blockchain technology represents an erosion of state 
power fits squarely within the literature on globalization. By contrast, state attempts to direct blockchain and related technologies, such as China's digitized renminbi, toward their domestic and international ends suggest they may be adapting to changing times. While some states move forward, it is worth asking if blockchain is indeed a viable tool of strategic competition. If so, how might we observe and measure states' strategic use of blockchain in global affairs?

The hackers behind Bitcoin not only had to design and build a technical solution to the problem of peer-to-peer electronic cash. They also had to create a system of rules to govern users' behaviors, for they could not rely on conventional institutions to do so. This thesis reminds developers and enthusiasts to consider the social implications of a technology once unleashed, for the technology may ultimately conform to the existing social structure it was intended to disrupt. Here too, political scientists would have much to say about constructing systems geared toward activism in cyberspace. If tools of surveillance and control can inspire equally powerful tools of secrecy and subversion, then might technology have an equalizing impact in state-society relations? Might blockchain technology, as a subversive technology, increase the effectiveness of transnational advocacy networks conducting affairs in illiberal regimes? Alternatively, can the suspicions between watchers and watched be resolved, grievances addressed, and reconciliation reached? Or is the tension between privacy and surveillance bound to escalate?

This thesis shows the Bitcoin network — and the people behind it - to be adaptive, flexible, and resilient. Its technical contributions remain to be fully applied, with prospects beyond payment systems in early development, such as distributed autonomous 
organizations, smart contracts, and blockchain voting. Most of all, the Bitcoin network exemplifies the possibility of an influential form of organization with no formal source of authority other than its code, no governmental or corporate sponsor, and at least at its outset, equality among peers. Perhaps that is a political success in its own right. 
References

Abrams, Rachel, and Nathaniel Popper. 2014. "Trading Site Failure Stirs Ire and Hope for Bitcoin.” The New York Times, February 25.

https://dealbook.nytimes.com/2014/02/25/trading-site-failure-stirs-ire-and-hopefor-bitcoin/.

Alferes, Jose Julio, Leopoldo Bertossi, Guido Governatori, Paul Fodor, and Dumitru Roman, eds. 2016. Rule Technologies: Research, Tools, and Applications. RuleML 2016. Lecture Notes in Computer Science, Vol. 9718. Basel: Springer International Publishing. https://doi.org/10.1007/978-3-319-42019-6.

Ammous, Saifedean. 2018. The Bitcoin Standard: The Decentralized Alternative to Centralized Money. Hoboken, NJ: John Wiley \& Sons.

Avdoshin, Sergey M., and Alex V. Lazarenko. 2018. "Bitcoin Users Deanonymization Methods." Proceedings of the Institute for System Programming of the Russian Academy of Sciences 30(1): 89-102. https://doi.org/10.15514/ispras-2018-30(1)$\underline{6}$.

Baker, Will. 2020. Bitcoin Kiosk [Photograph]. Portland, OR, April 24.

Bech, Morten and Rodney Garratt. 2017. "Central Bank Cryptocurrencies.” BIS Quarterly Review, September 17, 55-70. Basel: Bank for International Settlements. https://www.bis.org/publ/qtrpdf/r_qt1709f.htm.

Blinder, Alan S. 2013. "The Macroeconomic Policy Paradox: Failing by Succeeding." The Annals of the American Academy of Political and Social Science 650(1): 2646. https://doi.org/10.1177/0002716213493080.

Blockchain.com. 2019. "Bitcoin Charts \& Graphs.” September 20. https://www.blockchain.com/charts/.

Bitcoin Unlimited. 2015. "Articles of Federation." https://www.bitcoinunlimited.info/resources/BUarticles.pdf.

Bloomberg News. 2013. "China Bans Financial Companies from Bitcoin Transactions." Bloomberg, December 5. https://www.bloomberg.com/news/articles/2013-1205/china-s-pboc-bans-financial-companies-from-bitcoin-transactions.

Böhme, Rainer, Michael Brenner, Tyler Moore, and Matthew Smith, eds. Financial Cryptography and Data Security. FC 2014. Lecture Notes in Computer Science, Vol. 8438. Berlin: Springer. https://doi.org/10.1007/978-3-662-44774-1.

Bonneau, Joseph, Andrew Miller, Jeremy Clark, Arvind Narayanan, Joshua A. Kroll, and Edward W. Felten. 2015. "SoK: Research Perspectives and Challenges for Bitcoin 
and Cryptocurrencies." In 2015 IEEE Symposium on Security and Privacy, San Jose, CA, May 18-20, 104-121. https://doi.org/10.1109/sp.2015.14.

Bryanov, Kirill. 2019. "Blockchain and the City: New York State as a 'Tough' Model of Crypto Regulation." Cointelegraph, February 21. https://cointelegraph.com/news/blockchain-and-the-city-new-york-state-as-atough-model-of-crypto-regulation.

Carty, Victoria. 2015. Social Movements and New Technology. New York: Routledge.

Chen, Jia, and Xiaojin Ren. 2018. "PBOC Gets Tougher on Bitcoin.” China Daily, January 5. http://www.chinadaily.com.cn/a/201801/05/WS5a4eb4cba31008cf16da527c.html.

Christin, Nicolas. 2013. "Traveling the Silk Road: A Measurement Analysis of a Large Anonymous Online Marketplace.” In WWW 2013: Proceedings of the 22nd International Conference on World Wide Web, Rio de Janiero, Brazil, May 1317, 213-224. https://doi.org/10.1145/2488388.2488408.

Cohen, Benjamin J. 2008. "The International Monetary System: Diffusion and Ambiguity." International Affairs 84(3): 455-470. https://doi.org/10.1111/j.14682346.2008.00717.x.

CoinMarketCap. 2020. “Top 100 Cryptocurrencies by Market Capitalization.” May 22. https://coinmarketcap.com.

Coleman, Stephen, and Deen Freelon, eds. 2015. Handbook of Digital Politics. Northampton, MA: Edward Elgar Publishing. https://doi.org/10.4337/9781782548768.

Coveware. 2020. "Ransomware Costs Double in Q4 as Ryuk, Sodinokibi Proliferate." Coveware [blog], January 22. https://www.coveware.com/blog/2020/1/22/ransomware-costs-double-in-q4-asryuk-sodinokibi-proliferate.

cryptography-owner@metzdowd.com [pseud.].2020. “The Cryptography and Cryptography Policy Mailing List." https://www.metzdowd.com/mailman/listinfo/cryptography.

Demirgüç-Kunt, Asli, Leora Klapper, Dorothe Singer, Saniya Ansar, and Jake Hess. 2018. The Global Findex Database 2017: Measuring Financial Inclusion and the Fintech Revolution. Washington, DC: World Bank. http://hdl.handle.net/10986/29510.

Dewey, Taylor, Juliane Kaden, Miriam Marks, Shun Matsushima, and Beijing Zhu. 2012. The Impact of Social Media on Social Unrest in the Arab Spring. Stanford, CA: Stanford University, March 20. 
https://publicpolicy.stanford.edu/publications/impact-social-media-social-unrestarab-spring.

Digital Currency Initiative. 2020. "Why Does Blockchain Matter Today?” MIT Media Lab. https://dci.mit.edu/about.

Dimitri, Nicola. 2017. "Bitcoin Mining as a Contest." Ledger 2(September): 31-37. https://doi.org/10.5195/ledger.2017.96.

Dion-Schwarz, Cynthia, David Manheim, and Patrick B. Johnston. 2019. Terrorist Use of Cryptocurrencies: Technical and Organizational Barriers and Future Threats. Santa Monica, CA: RAND Corporation. https://doi.org/10.7249/rr3026.

Dyer, Larry. 1993. Rebels with a Cause [Cover photograph]. Wired, May/June. https://www.wired.com/story/wired-cover-browser-1993/.

Ethereum. 2019. "Build Unstoppable Blockchain Applications.” March 15. https://www.ethereum.org/.

Etherscripter. 2020. "What is Ethereum?" https://etherscripter.com/what is ethereum.html.

Facebook. 2020. "Facebook Reports First Quarter 2020 Results." PRNewswire, April 29. https://www.prnewswire.com/news-releases/facebook-reports-first-quarter-2020results-301049682.html.

FATF. 2014. Virtual Currencies: Key Definitions and Potential AML/CFT Risks. Paris: Financial Action Task Force, June. https://www.fatfgafi.org/media/fatf/documents/reports/Virtual-currency-key-definitions-andpotential-aml-cft-risks.pdf.

FATF. 2019. Guidance for a Risk-Based Approach to Virtual Assets and Virtual Asset Service Providers. Paris: Financial Action Task Force, June. https://www.fatfgafi.org/media/fatf/documents/recommendations/RBA-VA-VASPs.pdf.

Foley, Sean, Jonathan R. Karlsen, and Tālis J. Putniņš. 2019. "Sex, Drugs, and Bitcoin: How Much Illegal Activity is Financed through Cryptocurrencies?" The Review of Financial Studies 32(5): 1798-1853. https://doi.org/10.1093/rfs/hhz015.

Garcia-Navarro, Lulu. 2019. "New Campaign Would Allow Cryptocurrency Donations To Be Distributed to Venezuelans" National Public Radio, April 21. https://www.npr.org/2019/04/21/715616224/new-campaign-wouldallowcryptocurrency-donations-to-be-distributed-to-venezuela.

Gilmore, John. 1991. "Privacy, Technology, and the Open Society." In CFP'91: The First Conference on Computers, Freedom \& Privacy, Computer Professionals for 
Social Responsibility, Burlingame, CA, March 26-28.

http://cpsr.org/prevsite/conferences/cfp91/gilmore.html/.

Golumbia, David. 2016. The Politics of Bitcoin: Software as Right-Wing Extremism. Minneapolis, MN: University of Minnesota Press.

Google Trends. 2020. "Interest Over Time." Google. https://trends.google.com/trends/explore?date=all\&q=bitcoin.

Haber, Stuart and W. Scott Stornetta. 1991. "How to Time-Stamp a Digital Document." Journal of Cryptology 3(2): 99-111. https://doi.org/10.1007/bf00196791.

Harris, Bryan. 2018. "Sanctions-Hit North Korea Faces Crisis over Hard Currency." Financial Times, April 4. https://www.ft.com/content/db90592e-3876-11e8-8eeee06bde01c544.

Hemmings, John. 2020. "Reconstructing Order: The Geopolitical Risks in China's Digital Silk Road." Asia Policy 15(1): 5-21. https://doi.org/10.1353/asp.2020.0002.

Hobbes, Thomas. [1651] 1965. Leviathan, or The Matter, Forme, \& Power of a Common-Wealth Ecclesiasticall and Civill. London: Oxford University Press.

Houy, Nicolas. 2016. “The Bitcoin Mining Game.” Ledger 1(December): 53-68. https://doi.org/10.5195/ledger.2016.13.

Hughes, Eric. 1993. “A Cypherpunk's Manifesto." https://www.activism.net/cypherpunk/manifesto.html.

IMF. 2020. "Currency Composition of Official Foreign Exchange Reserves." IMF Data. Washington, DC: International Monetary Fund, March 31. https://data.imf.org/?sk=E6A5F467-C14B-4AA8-9F6D-5A09EC4E62A4.

Johnson, Benjamin, Aron Laszka, Jens Grossklags, Marie Vasek, and Tyler Moore. 2014. "Game-Theoretic Analysis of DDoS Attacks Against Bitcoin Mining Pools." In Financial Cryptography and Data Security. FC 2014. Lecture Notes in Computer Science, Vol. 8438, eds. Rainer Böhme, Michael Brenner, Tyler Moore, and Matthew Smith, 72-86. Berlin: Springer. https://doi.org/10.1007/978-3-66244774-1 6.

Kahler, Miles, ed. 2009. Networked Politics: Agency, Power, and Governance. Ithaca, NY: Cornell University Press.

Kahler, Miles. 2009. "Collective Action and Clandestine Networks: The Case of al Qaeda." In Networked Politics: Agency, Power, and Governance, ed. Miles Kahler, 103-126. Ithaca, NY: Cornell University Press. 
Kaminska, Izabella. 2017. "The HODL.” FT Alphaville, December 22. https://ftalphaville.ft.com/2017/12/22/2197074/the-hodl/.

Katz, Michael L. and Carl Shapiro. 1994. "Systems Competition and Network Effects." Journal of Economic Perspectives 8(2): 93-115. https://doi.org/10.1257/jep.8.2.93.

Krygier, Rachelle. 2018. "Venezuela Launches the 'Petro,' Its Cryptocurrency." The Washington Post, February 20. https://www.washingtonpost.com/news/worldviews/wp/2018/02/20/venezuelalaunches-the-petro-its-cryptocurrency/.

Lee Kuo Chuen, David, ed. 2015. Handbook of Digital Currency: Bitcoin, Innovation, Financial Instruments, and Big Data. San Diego: Elsevier. https://doi.org/10.1016/C2014-0-01905-3.

Leiner, Barry M., Vinton G. Cert, David D. Clark, Robert E. Kahn, Leonard Kleinrock, Daniel C. Lynch, Jon Postel, Larry G. Roberts, and Stephen Wolff. 1997. "Brief History of the Internet." Internet Society. https://www.internetsociety.org/internet/history-internet/brief-history-internet/.

Le Maire, Bruno. 2019. "Facebook's Libra is a Threat to National Sovereignty." Financial Times, October 17. https://www.ft.com/content/bf2f588e-ef63-11e9a55a-30afa498db1b.

Lessig, Lawrence. 1999. Code and Other Laws of Cyberspace. New York: Basic Books.

Levy, Steven. 2001. Crypto: How the Code Rebels Beat the Government-Saving Privacy in the Digital Age. New York: Penguin Books.

Lewenberg, Yoad, Yoram Bachrach, Yonaten Somplinsky, Aviv Zohar, and Jeffrey S. Rosenschein. 2015. "Bitcoin Mining Pools: A Cooperative Game Theoretic Analysis." In Proceedings of the 14th International Conference on Autonomous Agents and Multiagent Systems (AAMAS 2015), Istanbul, Turkey, May 4-8, 919927. https://academic.microsoft.com/paper/2263222003/.

Libra Association. 2019. “An Introduction to Libra.” Libra.org, June. https://libra.org/enUS/wp-content/uploads/sites/23/2019/06/LibraWhitePaper_en_US.pdf.

Liu, Ziyao, Nguyen Cong Luong, Wenbo Wang, Dusit Niyato, Ping Wang, Ying-Chang Liang, and Dong In Kim. 2019. “A Survey on Blockchain: A Game Theoretical Perspective.” IEEE Access 7(April): 47615-47643. https://doi.org/10.1109/access.2019.2909924.

Ludlow, Peter, ed. 2001. Crypto Anarchy, Cyberstates, and Pirate Utopias. Cambridge, MA: The MIT Press. https://doi.org/10.7551/mitpress/2229.001.0001. 
Ma, Adrian. 2019. “Ohio Experiments with Bitcoin Tax Payments.” Marketplace, April 8. https://www.marketplace.org/2019/04/08/ohio-experiments-bitcoin-taxpayments/.

Mahtani, Shibani, Gerry Shih, and Tiffany Liang. 2019. "With Threats and Propaganda, China Tries to Silence Support for Hong Kong Protests." The Washington Post, September 6. https://www.washingtonpost.com/world/asia pacific/with-threatsand-propaganda-china-tries-to-silence-support-for-hong-kongprotests/2019/09/06/72ab704c-cfb0-11e9-a620-0a91656d7db6 story.html.

Manne, Robert. 2011. "The Cypherpunk Revolutionary.” The Monthly, March. https://www.themonthly.com.au/issue/2011/february/1324596189/robertmanne/cypherpunk-revolutionary\#mtr.

May, Timothy C. 1992. "The Crypto Anarchist Manifesto." https://www.activism.net/cypherpunk/crypto-anarchy.html.

McAdam, Doug, Sidney Tarrow, and Charles Tilly. 2001. Dynamics of Contention. Cambridge: Cambridge University Press.

McCullagh, Declan. 2001. “Gay Site Halts Death 'Advice'.” Wired, December 1. https://www.wired.com/2001/12/gay-site-halts-death-advice/.

McNamara, Luke. "Why is North Korea So Interested in Bitcoin?" FireEye [blog], September 11. https://www.fireeye.com/blog/threat-research/2017/09/northkorea-interested-in-bitcoin.html.

Mougayar, William. 2016. "Explaining the Blockchain via A Google Docs Analogy." Startup Management, September 6. http://startupmanagement.org/2016/09/06/explaining-the-blockchain-via-agoogle-docs-analogy/.

Nakamoto, Satoshi (satoshi@vistomail.com). [pseud.]. 2008a. "Bitcoin P2P E-Cash Paper." The Cryptography Mailing List, October 31. https://www.metzdowd.com/pipermail/cryptography/2008-October/014810.html.

Nakamoto, Satoshi. [pseud.]. 2008b. "Bitcoin: A Peer-to-Peer Electronic Cash System," October 31. https://bitcoin.org/bitcoin.pdf.

Nakamoto, Satoshi (satoshin@gmx.com). [pseud.]. 2008c. Bitcoin.org, November 1. https://web.archive.org/web/20090131115053/http://bitcoin.org/.

Nakamoto, Satoshi. [pseud.]. 2009. "Bitcoin Open Source Implementation of P2P Currency." P2P Foundation Forum, February 15. http://p2pfoundation.ning.com/forum/topics/bitcoin-open-source. 
Odiboh, Oscar, Charity Ben-Enukora, Toluwalope Oresanya, Darlynton Yartey, and Ayoola Aiyelabola. 2017. "Awareness on Digital Security and E-Business in Nigeria." In International Conference on Education, Social Sciences and Humanities, Dubai, UAE, July 10-12, 1402-1408. http://eprints.covenantuniversity.edu.ng/id/eprint/10093.

Olson, Mancur. 1965. The Logic of Collective Action: Public Goods and the Theory of Groups. Cambridge, MA: Harvard University Press.

Orcutt, Mike. 2019. "China is About to Launch its Own Digital Currency. Here's What We Know So Far.” MIT Technology Review, September 13. https://www.technologyreview.com/f/614314/china-is-about-to-launch-its-owndigitalcurrency-heres-what-we-know-so-far/.

O’Sullivan, Andrea. 2017. "Ungoverned or Anti-Governance? How Bitcoin Threatens the Future of Western Institutions." Journal of International Affairs 71(2): 90-102. https://www.jstor.org/stable/26552331.

Pieters, Gina. 2016a. "Does Bitcoin Reveal New Information About Exchange Rates and Financial Integration?" Globalization and Monetary Policy Institute, Working Paper No. 292. Dallas, TX: Federal Reserve Bank of Dallas, December. https://doi.org/10.24149/gwp292.

Pieters, Gina. 2016b. "The Potential Impact of Decentralized Virtual Currency on Monetary Policy," Annual Report, Globalization and Monetary Policy Institute, 20-25. Dallas, TX: Federal Reserve Bank of Dallas. https://www.dallasfed.org/ /media/documents/institute/annual/2016/annual16d.pd f.

Pieters, Gina, and Sofia Vivanco. 2016. "Financial Regulations and Price Inconsistencies Across Bitcoin Markets." Globalization and Monetary Policy Institute, Working Paper No. 293. Dallas, TX: Federal Reserve Bank of Dallas, December. https://doi.org/10.24149/gwp293.

Popper, Nathaniel. 2015. Digital Gold: Bitcoin and the Inside Story of the Misfits and Millionaires Trying to Reinvent Money. New York: HarperCollins.

Rapoza, Kenneth. 2017. "Cryptocurrency Exchanges Officially Dead in China.” Forbes, November 2. http://www.forbes.com/sites/kenrapoza/2017/11/02/cryptocurrencyexchanges-officially-dead-in-china.

Ratna, Tavni. 2020. "Iran Has a Bitcoin Strategy to Beat Trump.” Foreign Policy, January 24. https://foreignpolicy.com/2020/01/24/iran-bitcoin-strategycryptocurrency-blockchain-sanctions/.

Rauchs, Michel, Apolline Blandin, Kristina Klein, Gina Pieters, Martino Recanatini, and Bryan Zhang. 2018. 2nd Global Cryptoasset Benchmarking Study. Cambridge, 
UK: Cambridge Centre for Alternative Finance, December. https://doi.org/10.2139/ssrn.3306125.

Reijers, Wessel, Fiachra O’Brolcháin, and Paul Haynes. 2016. "Governance in Blockchain Technologies and Social Contract Theories." Ledger 1(December): 134-151. https://doi.org/10.5195/ledger.2016.62.

Rousseau, Jean-Jacques. [1761]. A Discourse upon the Origin and Foundation of the Inequality among Mankind. London: R. and J. Dodsley. Eighteenth Century Collections Online. https://link.gale.com/apps/doc/CW0118502126/ECCO? $\mathrm{u}=$ oregon_portland\&sid= ECCO\&xid=2c6d2a 74 .

Samuelson, Paul A. 1954. "The Pure Theory of Public Expenditure." The Review of Economics and Statistics 36(4): 387-389. https://doi.org/10.2307/1925895.

Santander InnoVentures. 2015. "Fintech 2.0 Paper: Rebooting Financial Services." https://santanderinnoventures.com/wp-content/uploads/2015/06/The-Fintech-2-0Paper.pdf.

Schelling, Thomas C. 1971. "Dynamic Models of Segregation." Journal of Mathematical Sociology 1(2):143-186. https://doi.org/10.1080/0022250X.1971.9989794.

Schelling, Thomas C. 1978. Micromotives and Macrobehavior. New York: W.W. Norton \& Company.

Schulze, Elizabeth. 2019. “Cryptocurrencies Are 'Clearly Shaking the System,' IMF's Lagarde Says." CNBC, April 10. https://www.cnbc.com/2019/04/11/cryptocurrencies-fintech-clearly-shaking-thesystem-imfs-lagarde.html.

Snow, David A., Donatella della Porta, Bert Klandermans, and Doug McAdam, eds. 2013. The Wiley-Blackwell Encyclopedia of Social and Political Movements. Malden, MA: Wiley-Blackwell. https://doi.org/10.1002/9780470674871.

Stevenson, Betsey and Justin Wolfers. 2011. "Trust in Public Institutions over the Business Cycle." American Economic Review: Papers \& Proceedings 101(3): 281-287. https://doi.org/10.1257/aer.101.3.281.

Swan, Melanie. 2016. "Blockchain Temporality: Smart Contract Time Specifiability with Blocktime." In Rule Technologies: Research, Tools, and Applications. RuleML 2016. Lecture Notes in Computer Science, Vol. 9718, eds. Jose Julio Alferes, Leopoldo Bertossi, Guido Governatori, Paul Fodor, and Dumitru Roman, 184196. Basel: Springer International Publishing. https://doi.org/10.1007/978-3-31942019-6 12. 
Takemoto, Yoshifumi, and Sophie Knight. 2014. "Mt. Gox Files for Bankruptcy, Hit with Lawsuit." Reuters, February 28. https://www.reuters.com/article/us-bitcoinmtgoxbankruptcy/mt-gox-files-for-bankruptcy-hit-with-lawsuitidUSBREA1R0FX20140228.

Tarrow, Sidney G. 2011. Power in Movement: Social Movements and Contentious Politics. Cambridge: Cambridge University Press.

Tarrow, Sidney G. 2013. "Contentious Politics.” In The Wiley-Blackwell Encyclopedia of Social and Political Movements, eds. David A. Snow, Donatella della Porta, Bert Klandermans, and Doug McAdam, 266-270. Malden, MA: Wiley-Blackwell. https://doi.org/10.1002/9780470674871.wbespm051.

Tassev, Lubomir. 2018. "Study Finds Less Than 40\% of BTC Addresses Are Economically Relevant." Bitcoin.com, December 21.

https://news.bitcoin.com/study-finds-less-than-40-of-btc-addresses-areeconomically-relevant/.

Tilly, Charles and Lesley J. Wood. 2012. Social Movements, 1768-2012. London: Taylor \& Francis Group.

UNCTAD. 2017. Information Economy Report 2017: Digitalization, Trade and Development. New York: United Nations Conference on Trade and Development. https://doi.org/10.18356/3321e706-en.

United Nations Security Council, 1718 Sanctions Committee (DPRK) Panel of Experts. 2019. "Report of the Panel of Experts Established Pursuant to Resolution 1874 (2009)," S/2019/171, March 5. New York: United Nations Security Council. https://undocs.org/en/S/2019/171.

US Congress. House of Representatives. Committee on Financial Services. 2019. Hearing Memo: An Examination of Facebook and Its Impact on the Financial Services and Housing Sectors. October 18. 116th Cong. https://www.congress.gov/116/meeting/house/110136/documents/HHRG-116BA00-20191023-SD002.pdf.

Wang, Canhui, Xiaowen Chu, and Qin Yang. 2019 "Measurement and Analysis of the Bitcoin Networks: A View from Mining Pools.” ArXiv, February 21. https://arxiv.org/abs/1902.07549.

Werbach, Kevin. 2018. The Blockchain and the New Architecture of Trust. Cambridge, MA: The MIT Press. https://doi.org/10.7551/mitpress/11449.001.0001.

World Bank. 2020. "Individuals using the Internet (\% of population)." The World Bank Group. https://data.worldbank.org/indicator/IT.NET.USER.ZS. 
WTO. 2018. World Trade Report 2018: The Future of World Trade: How Digital Technologies are Transforming Global Commerce. Geneva: World Trade Organization. https://doi.org/10.30875/f309483f-en.

Xinhua. 2019. "Xi Stresses Development, Application of Blockchain Technology." China Daily, October 25. https://www.chinadaily.com.cn/a/201910/25/WS5db3001ca310cf3e35573b5e.htm 1 .

Yeow, Addy. 2020. “Global Nodes Distribution.” Bitnodes, May 12. https://bitnodes.io.

Yermack, David. 2015. "Is Bitcoin a Real Currency? An Economic Appraisal.” In Handbook of Digital Currency: Bitcoin, Innovation, Financial Instruments, and Big Data, ed. David Lee Kuo Chuen, 31-43. San Diego: Elsevier. https://doi.org/10.1016/b978-0-12-802117-0.00002-3.

Yin, Chien-Chung. 1998. "Equilibria of Collective Action in Different Distributions of Protest Thresholds." Public Choice 97(4): 535-567. https://www.jstor.org/stable/30024447.

Zhao, Yinan. 2020. “'Digital Currency for Cadres': A Communist Textbook Proves to Be a Hit." Bloomberg News, January 13. https://www.bloomberg.com/news/articles/2020-01-13/-digital-currency-forcadres-is-china-s-latest-how-to-book-hit. 